\title{
r-PROCESS LANTHANIDE PRODUCTION AND HEATING RATES IN KILONOVAE
}

\author{
JONAS LIPPUNER AND LUKE F. ROBERTS ${ }^{1}$ \\ TAPIR, Walter Burke Institute for Theoretical Physics, California Institute of Technology, MC 350-17, 1200 E California Boulevard, \\ Pasadena CA 91125, USA; jlippuner@tapir.caltech.edu \\ Received 2015 August 31; accepted 2015 November 4; published 2015 December 11
}

\begin{abstract}
r-process nucleosynthesis in material ejected during neutron star mergers may lead to radioactively powered transients called kilonovae. The timescale and peak luminosity of these transients depend on the composition of the ejecta, which determines the local heating rate from nuclear decays and the opacity. Kasen et al. and Tanaka \& Hotokezaka pointed out that lanthanides can drastically increase the opacity in these outflows. We use the new general-purpose nuclear reaction network SkyNet to carry out a parameter study of r-process nucleosynthesis for a range of initial electron fractions $Y_{\mathrm{e}}$, initial specific entropies $s$, and expansion timescales $\tau$. We find that the ejecta is lanthanide-free for $Y_{\mathrm{e}} \gtrsim 0.22-0.30$, depending on $s$ and $\tau$. The heating rate is insensitive to $s$ and $\tau$, but certain, larger values of $Y_{\mathrm{e}}$ lead to reduced heating rates, due to individual nuclides dominating the heating. We calculate approximate light curves with a simplified gray radiative transport scheme. The light curves peak at about a day (week) in the lanthanide-free (-rich) cases. The heating rate does not change much as the ejecta becomes lanthanide-free with increasing $Y_{\mathrm{e}}$, but the light-curve peak becomes about an order of magnitude brighter because it peaks much earlier when the heating rate is larger. We also provide parametric fits for the heating rates between 0.1 and 100 days, and we provide a simple fit in $Y_{\mathrm{e}}, s$, and $\tau$ to estimate whether or not the ejecta is lanthanide-rich.
\end{abstract}

Key words: gamma-ray burst: general - gravitational waves - nuclear reactions, nucleosynthesis, abundances stars: neutron

\section{INTRODUCTION}

The merger of a compact binary system that includes at least one neutron star, hence the merger of a neutron star with a black hole (NSBH) or the merger of two neutron stars (NSNS), is likely to eject a significant amount of material during the final stages of coalescence (Lattimer et al. 1977) in addition to emitting gravitational waves that may be observed by gravitational wave detectors such as advanced LIGO (The LIGO Scientific Collaboration 2015) and possibly powering short gamma-ray bursts (sGRBs; e.g., Lee \& Ramirez-Ruiz 2007; Nakar 2007; Gehrels et al. 2009). The material that is unbound during the merger is of interest for two main reasons. First, the majority of the mass ejected in these events is very neutron-rich. Once the material decompresses from initial densities close to nuclear density, the large number of neutrons can rapidly capture on the few heavy nuclides present and produce nuclei up to nuclear mass 300 . This process is called the r-process because neutrons are captured rapidly compared to the $\beta$-decay timescale of the unstable nuclides produced by neutron capture. Thus the r-process quickly creates heavy, very neutron-rich nuclides that eventually decay back to stability after the neutron capture ceases (Burbidge et al. 1957). Depending on the rate of NSBH and NSNS mergers and the amount of neutron-rich material ejected during these events, they can be the dominant source of r-process nucleosynthesis in the universe (Argast et al. 2004; Shen et al. 2014; RamirezRuiz et al. 2015; van de Voort et al. 2015).

Second, observable electromagnetic signals may be associated with these ejecta. A radio transient that occurs on a timescale of a few weeks can be powered by the interaction of the ejecta with the surrounding medium (Nakar \& Piran 2011). Additionally, radioactive decay of unstable nuclides formed during decompression of the ejecta can power a transient in the

\footnotetext{
${ }^{1}$ NASA Einstein Fellow.
}

optical or infrared that peaks on a timescale of a day to a week (Li \& Paczyński 1998; Kulkarni 2005; Metzger et al. 2010; Kasen et al. 2013; Tanaka \& Hotokezaka 2013). These are often referred to as either "kilonovae" (Metzger et al. 2010) or "macronovae"(Kulkarni 2005). In fact, one of these events may have been observed. An excess in the infrared afterglow of nearby GRB 130603B, which was an sGRB, has been interpreted by some authors as a strong indicator of a transient powered by the decay of r-process material (Berger et al. 2013; Tanvir et al. 2013). A similar kilonova like excess has recently been observed in the afterglow of GRB 060614 (Jin et al. 2015; Yang et al. 2015).

Although almost all of the ejected material will be neutronrich, there can be a significant spread in the electron fraction of this neutron-rich material. The composition will depend on whether the material was ejected tidally (Lattimer et al. 1977; Freiburghaus et al. 1999), dynamically from the region where the two neutron stars collide (Bauswein et al. 2013; Hotokezaka et al. 2013a), or from the accretion disk that forms after the merger (Fernández \& Metzger 2013; Perego et al. 2014; Just et al. 2015). Since the material ejected by all of these mechanisms starts out as cold, catalyzed material in a neutron star, the final electron fraction of the material depends on the weak interaction timescale relative to the dynamical timescale of the ejecta. If the temperature and local neutrino density are low, and therefore weak interactions are slow, the electron fraction is unaltered. This is the case for the tidal ejecta, which is predicted to have a very low electron fraction (Korobkin et al. 2012). Conversely, material ejected from the disk stays near the compact object for a long period and can achieve betaequilibrium at lower density and higher temperature (Just et al. 2015; Richers et al. 2015). The dynamical ejecta from the contact region sits somewhere in between, as it is ejected rapidly but shocked to high temperatures and irradiated 
strongly by neutrinos, which can significantly alter the initial electron fraction (Wanajo et al. 2014; Goriely et al. 2015).

At low initial electron fractions $\left(Y_{\mathrm{e}} \lesssim 0.2\right)$, the final composition of the ejecta is relatively insensitive to the initial electron fraction of the material because a strong r-process occurs and fission cycling produces a robust pattern (Metzger et al. 2010; Goriely et al. 2011; Roberts et al. 2011). But for higher electron fractions $\left(0.2 \lesssim Y_{\mathrm{e}} \lesssim 0.3\right)$, an incomplete r-process can occur and the composition will be much more sensitive to the properties of the outflow (Korobkin et al. 2012; Grossman et al. 2014; Kasen et al. 2015). In addition to the total mass and velocity of the ejecta, the composition of the ejecta at around a day-which determines the nuclear heating rate and opacity of the material-plays a large role in determining the properties of the kilonova (Li \& Paczyński 1998). Since losses due to adiabatic expansion rob all of the initial energy from the outflow, almost all of the energy that powers the transient must come from thermalizing the products of nuclear decay (Metzger et al. 2010). This in turn implies that the peak luminosity of a kilonova is sensitive to the composition.

The opacity of the material determines the timescale on which the ejecta becomes optically thin and therefore the timescale on which the transient will peak. Kasen et al. (2013) and Tanaka \& Hotokezaka (2013) have shown that continuum opacity is very sensitive to the presence of lanthanides, and possibly actinides, in the outflow. Due to their large atomic complexity, lanthanides and actinides have a very large number of lines relative to iron group elements and therefore their presence drastically increases the opacity of the material and causes predicted kilonovae to peak on timescales of around a week (Barnes \& Kasen 2013; Tanaka \& Hotokezaka 2013). Older models that assumed iron-like opacities predicted a peak timescale of around a day (Metzger et al. 2010; Goriely et al. 2011; Roberts et al. 2011). Significant lanthanide and actinide production requires very neutron-rich conditions, so Metzger \& Fernández (2014) have suggested that measurement of the peak time of a kilonova might provide insight into the composition of the outflow.

In this work, we present a parameter study of detailed nucleosynthesis calculations in NSBH or NSNS merger scenarios and their associated kilonova light curves. We focus in particular on the mass fraction of lanthanides and actinides present in the ejecta, the radioactive heating rate at 1 day, and how these properties depend on the initial conditions of the outflow. As expected, the lanthanide and actinide abundances depend strongly on the electron fraction, but the entropy and expansion timescale can also play an important role in certain cases. In contrast, we find that the nuclear decay heating rate does not depend as strongly on the initial electron fraction and it changes relatively smoothly when going from lanthanide-rich to lanthanide-free cases. The peak timescale, peak luminosity, and spectral temperature of our modeled kilonovae differ substantially due to the effect of the lanthanides and actinides on the opacity. In some cases, we also find very early and bright transients due to a neutron-rich freeze-out, which was proposed by Kulkarni (2005) and Metzger et al. (2015).

In Section 2, we describe our parametrized nucleosynthesis calculations and discuss how lanthanide production and the nuclear heating rate varies over our chosen parameter space. In Section 3, we present simplified kilonova light-curve models and examine how these transients vary with outflow properties.
We then conclude in Section 4. Lanthanides and actinides both have open $f$-shells and thus a similar valence electron structure, which means their impact on the opacity is similar (Kasen et al. 2013). Therefore, we will use the term "lanthanides" to refer to both lanthanides and/or actinides, unless otherwise noted.

\section{PARAMETERIZED EJECTA NUCLEOSYNTHESIS}

The details of the r-process abundance pattern, especially the position of the third peak, can be sensitive to the nuclear mass model, reaction rates, and fission fragment distributions that are used (e.g., Goriely et al. 2005; Arcones \& MartínezPinedo 2011; Mumpower et al. 2012; de Jesús Mendoza-Temis et al. 2014; Eichler et al. 2014). Here, we are less interested in the detailed final abundance patterns at high mass and more interested in the surfaces in our parameter space at which lanthanide production ceases. Therefore, we employ a single mass model and set of reaction rates. We use two models for fission fragments, but our main results are insensitive to this choice.

Rather than post-processing full hydrodynamic models as was done in Goriely et al. (2011), Korobkin et al. (2012), Grossman et al. (2014), Wanajo et al. (2014), Just et al. (2015), Martin et al. (2015), we use a parametrized approach that allows us to systematically study the impact of different ejecta properties on the properties of the ejected material relevant to kilonovae. Kasen et al. (2015) performed preliminary investigations of the electron fraction at which lanthanide production ceases, but they did not investigate how this influences the nuclear decay heating rate and only considered a small region of the parameter space.

We use the following three parameters to characterize the expanding material that undergoes r-process nucleosynthesis and produces a kilonova.

(i) The initial electron fraction $Y_{\mathrm{e}}=N_{\mathrm{p}} / N_{\mathrm{B}}$, where $N_{\mathrm{p}}$ is the total number of protons (free or inside nuclei) and $N_{\mathrm{B}}$ is the total number of baryons. We sample $Y_{\mathrm{e}}$ uniformly between 0.01 (very neutron-rich matter) and 0.5 (symmetric matter). We do not consider $Y_{\mathrm{e}}>0.5$ because the r-process requires a neutronrich environment.

(ii) The initial specific entropy $s$, which we sample logarithmically between 1 and $100 k_{\mathrm{B}}$ baryon $^{-1}$.

(iii) The expansion timescale $\tau$, which determines how fast the density decreases during nuclear burning. We sample $\tau$ logarithmically between 0.1 and $500 \mathrm{~ms}$. We choose an analytic density profile that initially decreases exponentially with time, i.e., $\rho \propto e^{-t / \tau}$, and then transitions smoothly to a homologous, $\rho \propto t^{-3}$, expansion. Requiring continuity of $\rho$ and $d \rho / d t$ fixes the matching point at $t=3 \tau$ and gives

$$
\rho(t)= \begin{cases}\rho_{0} e^{-t / \tau} & \text { if } t \leqslant 3 \tau, \\ \rho_{0}\left(\frac{3 \tau}{e t}\right)^{3} & \text { if } t \geqslant 3 \tau,\end{cases}
$$

where $\rho_{0}$ is the initial density and $e$ is Euler's number. This parameterization of the density is chosen because it gives us direct control over the dynamical timescale at the time of r-process nucleosynthesis but still matches smoothly to the density profile expected for homologous ejecta. We have also found that this profile gives a good approximation to density histories of Lagrangian fluid elements in the ejecta of BHNS 
mergers simulations (Foucart et al. 2014; M. Duez 2015, private communication)

We determine $\rho_{0}$ by setting the initial temperature to $T=6 \times 10^{9} \mathrm{~K}$ and then finding the density for which nuclear statistical equilibrium (NSE; with the given $Y_{\mathrm{e}}$ ) produces a set of abundances that has the prescribed initial entropy $s$. The entropy is calculated from the NSE distribution using a modified version of the Helmholtz equation of state (EOS) based on Timmes \& Swesty (2000). The EOS has been modified to calculate the entropy for each nuclear species separately, rather than using average mass and charge numbers, and it also includes the internal partition functions of all nuclear species, which we obtained from the WebNucleo database distributed $^{2}$ with REACLIB (see below). The resulting initial densities range from $7.1 \times 10^{5}$ to $1.4 \times 10^{12} \mathrm{~g} \mathrm{~cm}^{-3}$.

Given $Y_{\mathrm{e}}, s$, and $\tau$, NSE determines $\rho_{0}$ (and thus $\rho(t)$ ) and the initial abundances. We then use the newly developed nuclear reaction network SkyNet for the abundance evolution. SkyNet is a general-purpose, modular nuclear reaction network that keeps track of entropy and temperature changes due to the nuclear reactions it is evolving. A detailed code description of the functionality and features of SkyNet is forthcoming (J. Lippuner $\&$ L. F. Roberts 2015, in preparation), and the source code will be publicly released together with that paper. In the meantime, anyone who wishes to use SkyNet can contact the authors and request access to the code.

We run SkyNet with nuclear reaction rates from the JINA REACLIB database ${ }^{3}$ (Cyburt et al. 2010). The nuclear data (masses and partition functions) were taken from the associated WebNucleo XML file distributed with REACLIB. Although REACLIB includes inverse rates for the strong reactions, SkyNet calculates these inverse rates from detailed balance, so that the rates are consistent with NSE. We also include different sets of spontaneous and neutron-induced fission rates, as REACLIB does not presently include any fission reactions. There are three sets of symmetric neutron-induced fission reactions: sym0, sym2, and sym 4, which produce 0,2 , and 4 free neutrons, respectively, for each fission event. There is also a set nonsym of non-symmetric fission reactions that do not produce any free neutrons. Each nucleosynthesis calculation includes one of the four neutron-induced fission reaction sets and the spontaneous fission reaction set. All the fission reactions and their rates are taken from the same sources used in Roberts et al. (2011).

We use beta-decay and electron capture rates from Fuller et al. (1982), Oda et al. (1994) and Langanke \& MartínezPinedo (2000) whenever they are available. For nuclei for which these rates are not available, the effects of electron blocking and positron capture are approximately included by assuming that only a ground state to ground state transition occurs as described in Arcones et al. (2010). These rates are then normalized such that they are equal to the vacuum decay rates given in REACLIB at low temperature and density, which

\footnotetext{
2 https://groups.nscl.msu.edu/jina/reaclib/db/library.php? action $=$ viewsnapshots

3 At the time of writing, the latest REACLIB snapshot (2013 April 02) contains 83 incorrect $\beta$-decay rates, which we corrected for this study. It appears that some lower limits of the half-lives published in the Nuclear Wallet Cards (http://www.nndc.bnl.gov/wallet) were put into REACLIB, but those lower limits can be very far away from realistic estimates of the half-lives. For example, REACLIB gives a half-life of $300 \mathrm{~ns}$ for ${ }^{216} \mathrm{~Pb}$ because the Nuclear Wallet Cards state the half-life is " > 300 ns," but Möller et al. (2003) gives a half-life of about $850 \mathrm{~s}$, which is much closer to the half-lives of similar nuclides.
}

Table 1

Parameter Values at Grid Points

\begin{tabular}{|c|c|c|c|c|c|}
\hline \multicolumn{3}{|c|}{ Low-resolution Points ${ }^{a}$} & \multicolumn{3}{|c|}{ Additional High-resolution Points ${ }^{\mathrm{b}}$} \\
\hline$Y_{\mathrm{e}}$ & $\begin{array}{c}s \\
\left(k_{\mathrm{B}} \text { baryon }^{-1}\right)\end{array}$ & $\begin{array}{c}\tau \\
(\mathrm{ms})\end{array}$ & $Y_{\mathrm{e}}$ & $\begin{array}{c}s \\
\left(k_{\mathrm{B}} \text { baryon }^{-1}\right)\end{array}$ & $\begin{array}{c}\tau \\
(\mathrm{ms})\end{array}$ \\
\hline \multirow[t]{2}{*}{0.01} & 1.0 & 0.10 & & & \\
\hline & & & 0.04 & 1.3 & 0.17 \\
\hline \multirow[t]{2}{*}{0.07} & 1.8 & 0.29 & & & \\
\hline & & & 0.10 & 2.4 & 0.49 \\
\hline \multirow[t]{2}{*}{0.13} & 3.2 & 0.84 & & & \\
\hline & & & 0.16 & 4.2 & 1.4 \\
\hline \multirow[t]{2}{*}{0.19} & 5.6 & 2.4 & & & \\
\hline & & & 0.22 & 7.5 & 4.2 \\
\hline \multirow[t]{2}{*}{0.25} & 10 & 7.1 & & & \\
\hline & & & 0.29 & 13 & 12 \\
\hline \multirow[t]{2}{*}{0.32} & 18 & 21 & & & \\
\hline & & & 0.35 & 24 & 35 \\
\hline \multirow[t]{2}{*}{0.38} & 32 & 59 & & & \\
\hline & & & 0.41 & 42 & 100 \\
\hline \multirow[t]{2}{*}{0.44} & 56 & 170 & & & \\
\hline & & & 0.47 & 75 & 290 \\
\hline 0.50 & 100 & 500 & & & \\
\hline
\end{tabular}

Notes.

a The low-resolution runs of the entire parameter space use only these grid points.

${ }^{\mathrm{b}}$ For the high-resolution runs of the entire parameter space we double the number of grid points. The high-resolution runs include the grid points shown in this column in addition to the the same points as the low-resolution runs.

can be thought of as setting the effective matrix element for the ground state to ground state transition. Because this procedure assumes a maximal $Q$-value for these weak rates, this provides a lower limit on the effect of the surrounding medium on the combined beta-decay and lepton capture rate. For this study, we run SkyNet with 7843 nuclear species, ranging up to $Z=112$ and $A=337$, and 110,793 nuclear reactions.

\subsection{Parameter Space}

We use a $9 \times 9 \times 9$ grid to cover the entire parameter space and run SkyNet for each point with all four sets of neutroninduced fission reactions (sym0, sym2, sym4, nonsym). We also run the sym0 fission reactions with a finer $17 \times 17 \times 17$ grid. The parameter values at the grid points are shown in Table 1 . The different fission reactions only result in small quantitative and no qualitative differences. Thus we only discuss and show plots of the high-resolution sym0 runs. Finally, we carry out a set of runs with high $Y_{\mathrm{e}}$ resolution ( $\Delta \quad Y_{\mathrm{e}}=0.005 \quad$ resulting in $99 \quad Y_{\mathrm{e}}$ points) for $s=1,10,30,100 k_{\mathrm{B}}$ baryon $^{-1}$ and $\tau=0.1,1,10 \mathrm{~ms}$ with the sym0 fission reactions. The data underlying all the results shown and discussed here (nucleosynthesis results, heating rate fit coefficients, light-curve model results, and integrated fractional heating contributions of all nuclides) are available at http://stellarcollapse.org/lippunerroberts2015.

Figure 1 shows the final abundances of a few selected cases, which span the whole range of $Y_{\mathrm{e}}$ and $s$ at intermediate values of the other two parameters. For the $s=10 k_{\mathrm{B}}$ baryon $^{-1}$ and $\tau=7.1 \mathrm{~ms}$ trajectories (left panel of Figure 1), the full r-process up to the third peak $(A \sim 190)$ for $Y_{\mathrm{e}}=0.01$ and $Y_{\mathrm{e}}=0.19$ is produced. We note good agreement of the second, third, and rare-Earth peak positions with the solar r-process 


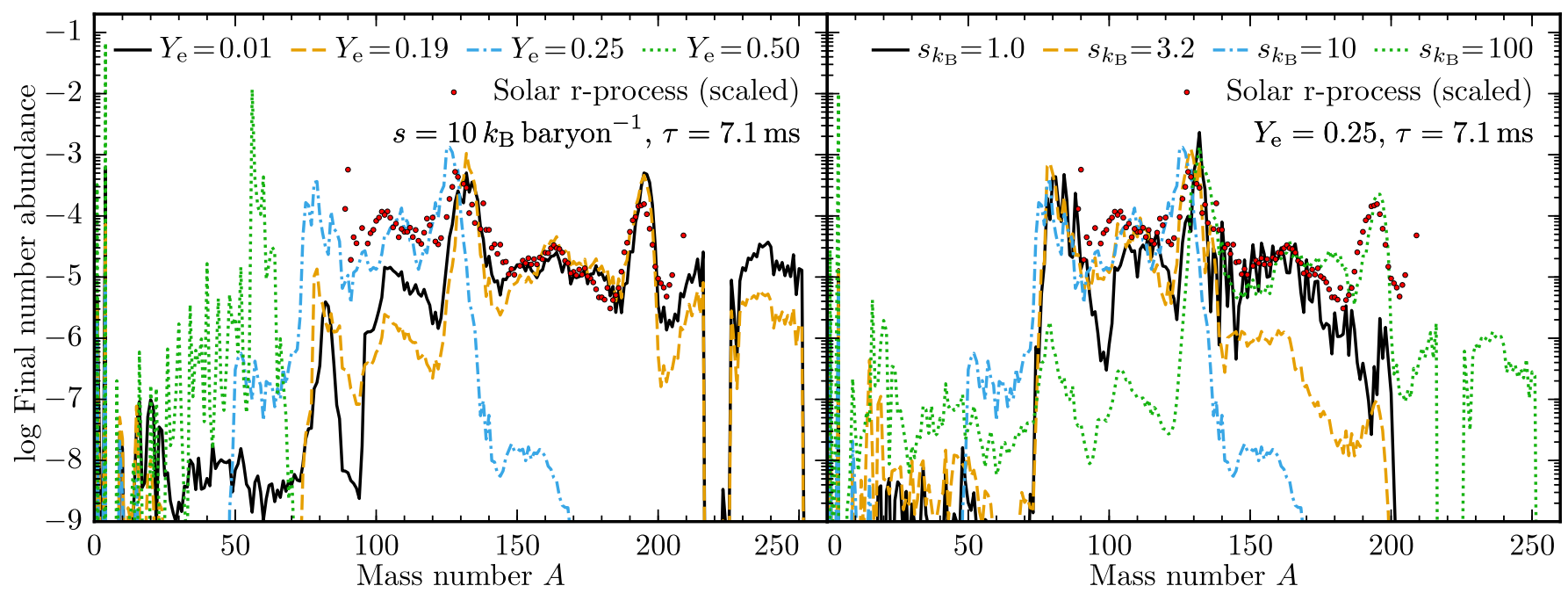

Figure 1. Final abundances of some selected nucleosynthesis calculations. Left: $Y_{\mathrm{e}}=0.01,0.19,0.25,0.50, s=10 k_{\mathrm{B}}$ baryon $^{-1}$, and $\tau=7.1 \mathrm{~ms}$. The full r-process is made, with substantial amounts of lanthanides and actinides, for $Y_{\mathrm{e}}=0.01$ and $Y_{\mathrm{e}}=0.19$. The $Y_{\mathrm{e}}=0.25$ trajectory is neutron-rich enough to make the second r-process peak, but not the third and not a significant amount of lanthanides. In the symmetric case $\left(Y_{\mathrm{e}}=0.5\right)$, mostly ${ }^{4} \mathrm{He}$ and iron-peak elements are produced. Right: $Y_{\mathrm{e}}=0.25, s=1.0,3.2,10,100 k_{\mathrm{B}}$ baryon $^{-1}$, and $\tau=7.1 \mathrm{~ms}$. With $s=1 k_{\mathrm{B}}$ baryon ${ }^{-1}$ a jagged r-process is obtained because there are only few free neutrons per seed nucleus available and nuclides with even neutron numbers are favored. Even though there are not many free neutrons available, there is still a significant amount of lanthanides in the $s=1 k_{\mathrm{B}}$ baryon $^{-1}$ case because the initial seed nuclei are very heavy. At higher entropies, the initial seeds become lighter and the initial free neutron abundance increases. However, the increase in the initial free neutron abundance is not enough to offset the decrease in the initial mass of the seeds and so we obtain a less complete r-process. The situation is reversed at $s=100 \mathrm{kB}_{\mathrm{B}}$ baryon $^{-1}$, where there is a very high neutron-to-seed ratio. In that case, a significant fraction of $\alpha$ particles are also captured on the seed nuclei. This leads to a full r-process in the $s=100 k_{\mathrm{B}}$ baryon $^{-1}$ case. $^{2}$

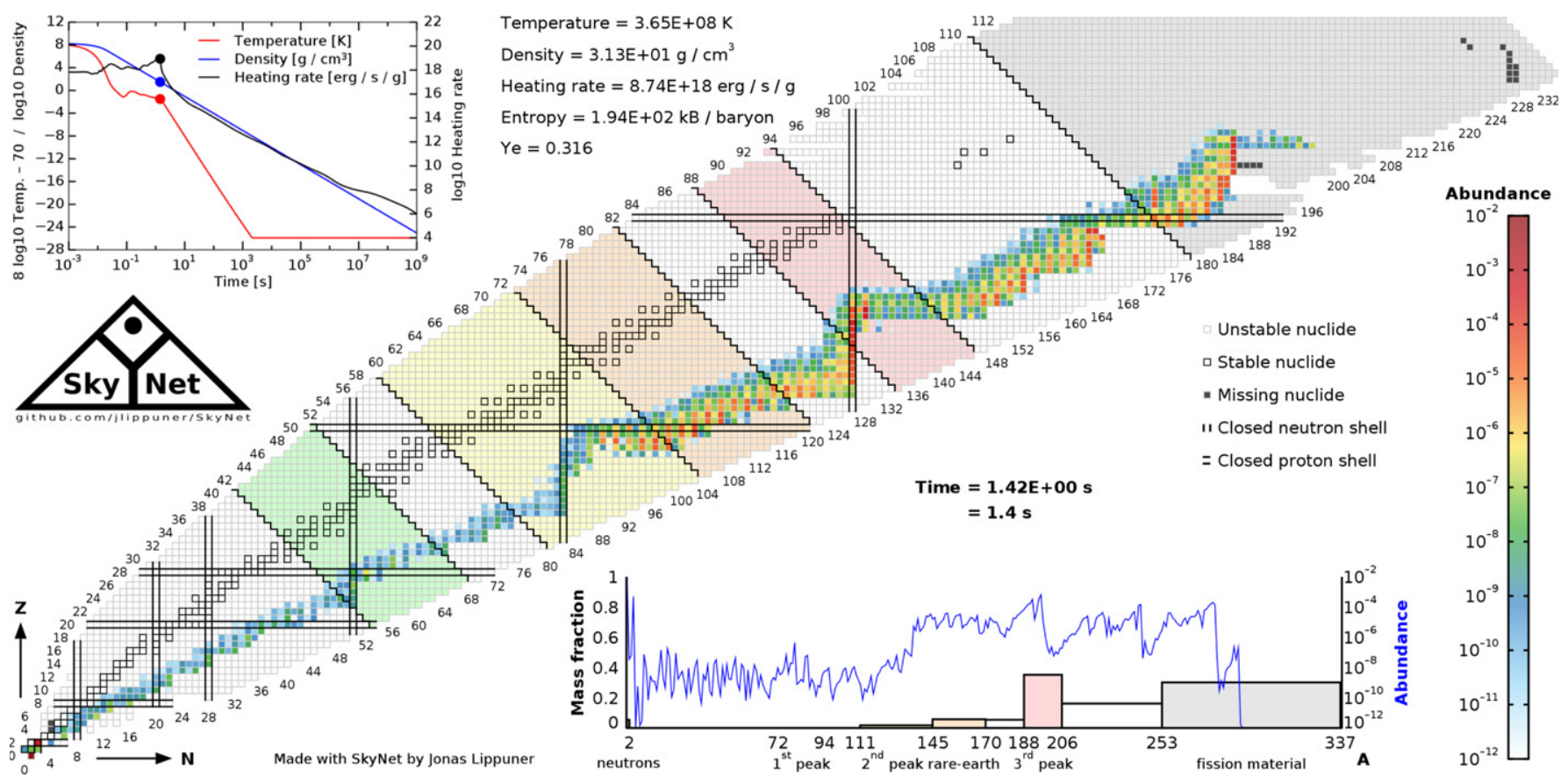

Figure 2. Frame from the animation of the nucleosynthesis calculation for $Y_{\mathrm{e}}=0.01, s=10 k_{\mathrm{B}}$ baryon $^{-1}$, and $\tau=7.1 \mathrm{~ms}$. The frame shows the full extent of the r-process just when free neutrons get exhausted. The plot in the upper left corner shows the temperature, density, and heating rate as function of time. The colored bands in the chart of nuclides correspond to the mass bins in the histogram at the bottom. The histogram shows the mass fractions on a linear scale while the blue curve shows the abundances as a function of mass on a logarithmic scale. The full animations are available at http://stellarcollapse.org/lippunerroberts2015.

abundances, although the third peak is slightly overproduced relative to the second peak. The abundance patterns of $Y_{\mathrm{e}}=0.01$ and $Y_{\mathrm{e}}=0.19$ are very similar because both cases are neutron-rich enough to produce nuclides with $A \gtrsim 250$, which eventually undergo fission. As the ejecta becomes less neutron-rich $\left(Y_{\mathrm{e}}=0.25\right.$ and $\left.Y_{\mathrm{e}}=0.50\right)$, the full $\mathrm{r}$-process is no longer produced; there are not enough neutrons available per seed nucleus to reach the third peak. At $Y_{\mathrm{e}}=0.25$, the first and second r-process peaks are produced. The right panel of Figure 1 shows the final abundances of cases with $Y_{\mathrm{e}}=0.25$, $\tau=7.1 \mathrm{~ms}$, and different initial entropies. Here, the electron fraction is too high to get to the third r-process peak at most entropies (all the cases with entropies between 10 and $75 k_{\mathrm{B}}$ baryon $^{-1}$ have virtually identical final abundances as the $s=10 k_{\mathrm{B}}$ baryon $^{-1}$ case). At $s=100 k_{\mathrm{B}}$ baryon $^{-1}$ the third $r$-process peak is obtained because the initial composition 
contains few seed nuclei and alpha particles are unable to efficiently combine to produce seed nuclei. Thus, the neutronto-seed ratio is significantly enhanced.

Animations of the full nucleosynthesis calculations for all seven cases shown in Figure 1 are available at http:// stellarcollapse.org/lippunerroberts2015. Figure 2 shows a frame from one of the animations.

\subsection{Lanthanide Turnoff and Heating Rate as a Function of $Y_{\mathrm{e}}$}

Figure 3 shows the final lanthanide and actinide mass fractions $X_{\mathrm{La}}$ and $X_{\mathrm{Ac}}$, respectively, along with the neutron mass fraction $X_{\mathrm{n}}$ at 10 minutes, which is the mean lifetime of a free neutron. Also shown is $\bar{A}_{\text {fin }}$, which is an estimate of the final average mass number $A$ of the material. It is defined as

$$
\bar{A}_{\text {fin }}=\frac{1}{Y_{\text {seed }}(0)+Y_{\alpha}(0) / 18},
$$

where $Y_{\alpha}(0)$ is the initial $\alpha$-particle abundance and $Y_{\text {seed }}(0)$ is the initial seed abundance (sum of abundances of all nuclides with $A \geqslant 12$ ). Since the $\alpha$-process ceases around $\mathrm{Kr}$ in neutron rich conditions (Woosley \& Hoffman 1992), it takes around 18 $\alpha$ particles to make a seed nucleus. Therefore, the quantity in the denominator of Equation (2) is approximately the number abundance of heavy nuclei present at the end of the r-process. We then arrive at Equation (2) by assuming that the total mass fraction of heavy nuclei at the end of the calculation is unity. Clearly, this assumption breaks down if there is fission cycling because then the number of seeds at the end is much larger than the number of initial seeds plus those produced by the $\alpha$ process. However, we are interested in the value of $\bar{A}_{\text {fin }}$ at the actinide and lanthanide turnoff, which preclude significant fission cycling because fission cycling only happens if nuclides heavier than actinides are produced, and so there is no problem in using the definition in Equation (2). At low electron fractions, $\alpha$-rich freeze-out does not occur due to the low initial abundance of $\alpha$ particles. We emphasize that $\bar{A}_{\text {fin }}$ only depends on the initial abundances, and thus it is useful to determine whether a certain trajectory is likely to produce large quantities of lanthanides or actinides, without having to perform any nucleosynthesis calculation.

Table 2 shows the values of $Y_{\mathrm{e}}$ and $\bar{A}_{\text {fin }}$ at which lanthanide and actinide production ceases (mass fraction goes below $\left.10^{-3}\right)$. In other words, if $Y_{\mathrm{e}}$ is lower than or $\bar{A}_{\text {fin }}$ larger than what is shown in Table 2 , then $X_{\mathrm{La}} \geqslant 10^{-3}$ or $X_{\mathrm{Ac}} \geqslant 10^{-3}$. The lanthanide turnoff is at $\bar{A}_{\text {fin }} \sim 100$ and the actinide turnoff is at $\bar{A}_{\text {fin }} \sim 130$. The cases where $X_{\mathrm{La}}<10^{-3}$ or $X_{\mathrm{Ac}}<10^{-3}$ for all $Y_{\mathrm{e}}$ are denoted by "-_" in Table 2, and they correspond to the strong neutron-rich freeze-outs in Figure 3, which means that the r-process did not happen (or at least not efficiently) in those cases because after about 10 minutes we are just left with free neutrons that will now decay to protons. In the case $s 10 \tau 0.1$ (which stands for $s=10 k_{\mathrm{B}}$ baryon $^{-1}$ and $\tau=0.1 \mathrm{~ms}$ ) where lanthanides are made, but no actinides above a mass fraction of $10^{-3}$, we see a weaker neutron-rich freeze-out in Figure 3. The neutron-rich freeze-outs happen at high initial entropies and short expansion timescales, where the ejecta is very hot and expands quickly, which leaves little time for neutrons to capture on seed nuclides. There is also a neutron-rich freeze-out in $s 30 \tau 1$ and $s 100 \tau 1$ models, but the freeze-out is weak enough to allow lanthanides and actinides to be produced, albeit in lower quantities. Metzger et al. (2015) suggested that a kilonova containing some mass with such short dynamical timescales could be preceded by an ultraviolet transient powered by these frozen-out neutrons.

Figure 3 shows that the heating rate from decay at 1 day is quite insensitive to $Y_{\mathrm{e}}$ at $Y_{\mathrm{e}} \lesssim 0.35$ and also fairly insensitive to the amount of lanthanides and actinides produced. As long as $X_{\mathrm{La}+\mathrm{Ac}}$ is more or less constant as a function of $Y_{\mathrm{e}}, M \epsilon$ at 1 day is also fairly constant. When the lanthanides turn off, there is a small bump in the heating rate in most cases and at larger $Y_{\mathrm{e}}$, after lanthanides have completely gone away, the heating rate drops only slightly (an order of magnitude or less). One might expect a larger decline of the heating rate once the full r-process stops happening, because the material is less neutronrich overall, more stable nuclei are produced directly, and thus the total radioactive decay energy should be lower. This is indeed true and we verified it by looking at the integrated nuclear heating amount as a function of $Y_{\mathrm{e}}$ (for fixed $s$ and $\tau$ ). We find that in most cases the total amount of heating drops by 1.5-2 orders of magnitude as $Y_{\mathrm{e}}$ goes from low values to high values. There is a smaller drop in the heating rates shown in Figure 3 , because there we only plot the instantaneous heating rate at 1 day. Since the $\beta$-decay energy is correlated with the decay timescale, we always see a similar instantaneous decay rate at the same point in time, as long as we have a collection of nuclides with half-lives at around a day. The picture changes at $Y_{\mathrm{e}} \gtrsim 0.35$ because there the final composition is dominated by one or a few individual nuclides, as opposed to a large ensemble of nuclides, which then determine the heating rate. This is discussed in detail in Section 2.4.

Since our parameter space is three-dimensional, we can go beyond giving a simple $Y_{\mathrm{e}}$ cutoff for lanthanide production. We use a heuristic method to fit for the coefficients of three inequalities in $Y_{\mathrm{e}}, \ln s$, and $\ln \tau$ that separate the lanthanide-rich and lanthanide-free regions of the parameter space. We find that

$$
\begin{aligned}
& X_{\mathrm{La}+\mathrm{Ac}} \geqslant 10^{-3} \text { if and only if } \\
& -1.00 Y_{\mathrm{e}}-0.00744 \ln s_{k_{\mathrm{B}}}+0.000638 \ln \tau_{\mathrm{ms}}+0.259 \geqslant 0, \\
& \quad \text { and } \\
& -0.990 Y_{\mathrm{e}}+0.117 \ln s_{k_{\mathrm{B}}}-0.0783 \ln \tau_{\mathrm{ms}}+0.452 \geqslant 0, \\
& \quad \text { and } \\
& -0.799 Y_{\mathrm{e}}-0.288 \ln s_{k_{\mathrm{B}}}+0.528 \ln \tau_{\mathrm{ms}}+1.88 \geqslant 0,
\end{aligned}
$$

where $s_{k_{\mathrm{B}}}$ is the entropy $s$ in units of $k_{\mathrm{B}}$ baryon $^{-1}$ and $\tau_{\mathrm{ms}}$ is the expansion timescale $\tau$ in units of milliseconds. The above statement only fails for 97 out of 4913 points in our parameter space, i.e., it is true for $98 \%$ of the parameter space. Most of the points where the above fails are very close to one of the planes, but there are a few points further away from the boundaries that fail too. Those points are all at very low $Y_{\mathrm{e}}$, high entropy, and very short expansion timescale, where we get strong neutronrich freeze-out. The results of the full parameter space are discussed in detail in Section 2.4.

\subsection{Fission Cycling}

If the r-process is strong enough to produce nuclides with masses near 300, these nuclides fission and the fission products then capture more neutrons, eventually getting up to $A \sim 300$ and fissioning again, creating a fission cycle. Thus fission cycling limits the maximum mass of nuclides produced in the 


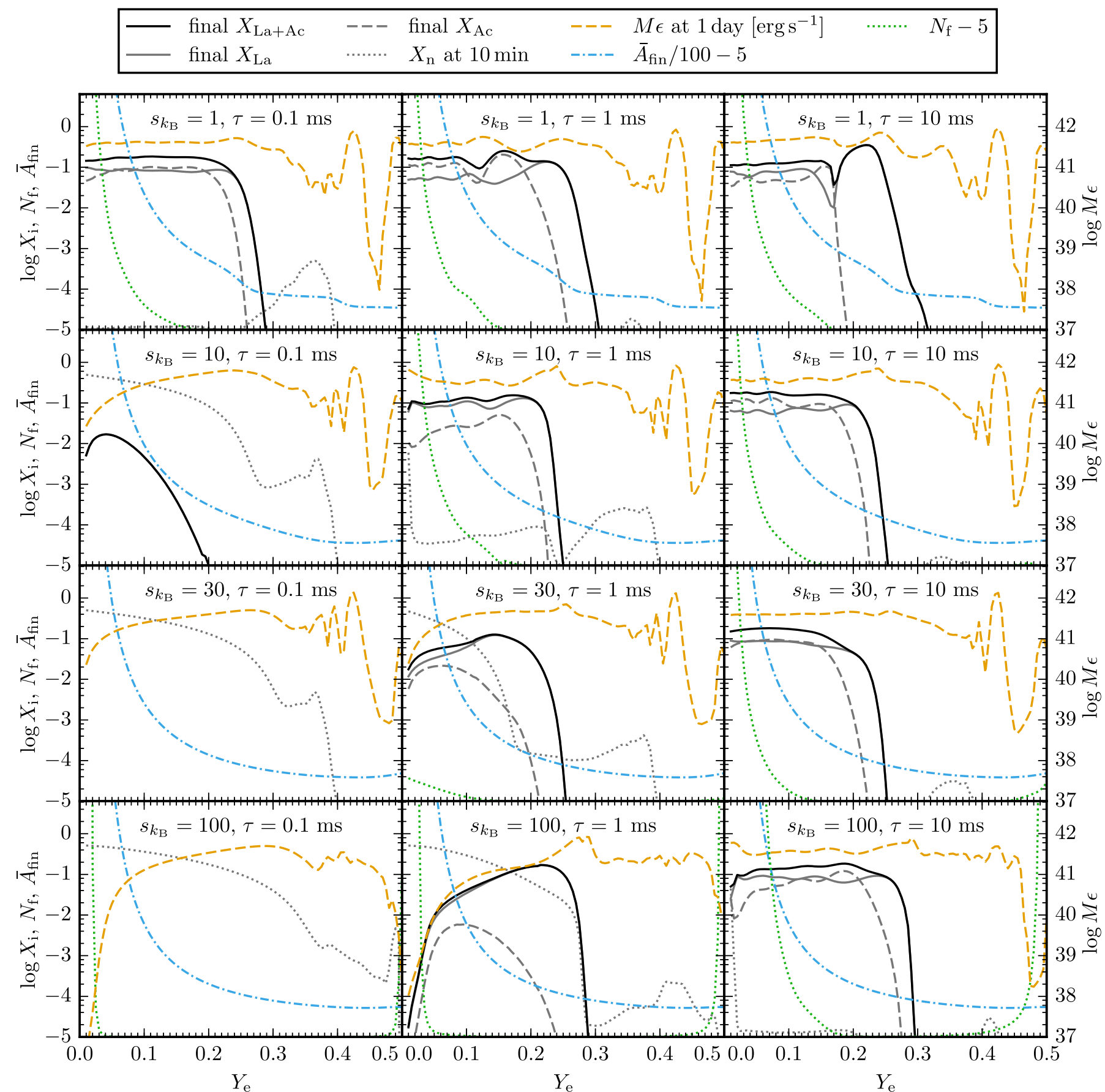

Figure 3. Results of the high-resolution $Y_{\mathrm{e}}$ runs. The lanthanide and actinide mass fractions, $X_{\mathrm{La}}$ and $X_{\mathrm{Ac}}$, and their sum, $X_{\mathrm{La}+\mathrm{Ac}}$, are fairly constant up to some critical value of $Y_{\mathrm{e}}$ in most cases because of fission cycling. The neutron abundance $X_{\mathrm{n}}$ at 10 minutes (the mean lifetime of a free neutron) is an indicator for a neutron-rich freeze-out, which occurs at high initial entropies and short expansion timescales, where the neutrons do not have time to capture on the seed nuclei. The heating rate $M \epsilon$ at 1 day with $M=10^{-2} M_{\odot}$ is fairly insensitive to $Y_{\mathrm{e}}$, except at high electron fractions $\left(Y_{\mathrm{e}} \gtrsim 0.4\right)$ where some individual nuclides start to dominate the heating. The estimated final average mass number $\bar{A}_{\mathrm{fin}}$ falls off monotonically with $Y_{\mathrm{e}}$ in all cases except $s=100 k_{\mathrm{B}}$ baryon $^{-1}$, where it rebounds at $Y_{\mathrm{e}}$ very close to 0.5 . There, the number of seed nuclei decreases drastically because $\alpha$-particles are initially produced in higher quantities, which increases the neutron-to-seed ratio. In those cases, the predicted number of fission cycles $N_{\mathrm{f}}$ is artificially increased at high $Y_{\mathrm{e}}$, because of production of seed nuclei by the triple- $\alpha$ process. Where Equation (3) accurately predicts the number of fission cycles, $N_{\mathrm{f}}$ falls off rapidly with $Y_{\mathrm{e}}$ and the point where it becomes zero is correlated with the actinide turnoff, because actinides are at the low end of the fissionable material mass range. Note that we plot $\bar{A}_{\text {fin }}$ and $N_{\mathrm{f}}$ on linear scales rather than log scales as all the other quantities. Also, we added a negative offset of 5 to both $\bar{A}_{\text {fin }}$ and $N_{\mathrm{f}}$ and we scaled $\bar{A}_{\text {fin }}$ by $1 / 100$ so that they fit onto our left vertical axis.

r-process, which washes out the initial conditions of the ejecta and hence the final abundances are determined by nuclear physics rather than the properties of the outflow.

The quantity $N_{\mathrm{f}}$ shown in Figure 3 is an estimate for the number of fission cycles that occurred during nucleosynthesis.
It is defined as

$$
N_{\mathrm{f}}=\frac{Y_{\text {seed }}\left(t=t_{n}\right)}{Y_{\text {seed }}(t=0)}-1
$$


Table 2

$\bar{A}_{\text {fin }}$ and $Y_{\mathrm{e}}$ at Lanthanide and Actinide Turnoff

\begin{tabular}{|c|c|c|c|c|c|}
\hline \multirow[b]{2}{*}{$\begin{array}{l}s \\
\left(k_{\mathrm{B}} \text { baryon }^{-1}\right)\end{array}$} & \multirow[b]{2}{*}{$\begin{array}{c}\tau \\
(\mathrm{ms})\end{array}$} & \multicolumn{2}{|c|}{$\begin{array}{c}\text { Lanthanide } \\
\text { turnoff }^{\mathrm{a}}\end{array}$} & \multicolumn{2}{|c|}{ Actinide turnoff ${ }^{a}$} \\
\hline & & $Y_{\mathrm{e}}$ & $\bar{A}_{\text {fin }}$ & $Y_{\mathrm{e}}$ & $\bar{A}_{\text {fin }}$ \\
\hline 1.0 & 0.1 & 0.27 & 94 & 0.25 & 123 \\
\hline 1.0 & 1 & 0.28 & 91 & 0.24 & 137 \\
\hline 1.0 & 10 & 0.28 & 93 & 0.18 & 192 \\
\hline 1.8 & 0.1 & 0.25 & 106 & 0.21 & 123 \\
\hline 1.8 & 1 & 0.27 & 100 & 0.21 & 125 \\
\hline 1.8 & 10 & 0.27 & 98 & 0.17 & 170 \\
\hline 3.0 & 0.1 & 0.23 & 118 & 0.20 & 135 \\
\hline 3.0 & 1 & 0.25 & 111 & 0.21 & 130 \\
\hline 3.0 & 10 & 0.27 & 106 & 0.18 & 150 \\
\hline 5.6 & 0.1 & 0.22 & 135 & 0.14 & 196 \\
\hline 5.6 & 1 & 0.23 & 127 & 0.21 & 138 \\
\hline 5.6 & 10 & 0.24 & 124 & 0.21 & 140 \\
\hline 10 & 0.1 & 0.13 & 223 & - & - \\
\hline 10 & 1 & 0.24 & 121 & 0.21 & 139 \\
\hline 10 & 10 & 0.24 & 120 & 0.21 & 139 \\
\hline 18 & 0.1 & - & - & - & - \\
\hline 18 & 1 & 0.24 & 102 & 0.20 & 130 \\
\hline 18 & 10 & 0.24 & 102 & 0.21 & 125 \\
\hline 30 & 0.1 & - & - & - & - \\
\hline 30 & 1 & 0.24 & 93 & 0.18 & 132 \\
\hline 30 & 10 & 0.24 & 93 & 0.20 & 113 \\
\hline 56 & 0.1 & - & - & - & - \\
\hline 56 & 1 & 0.24 & 94 & 0.16 & 143 \\
\hline 56 & 10 & 0.24 & 94 & 0.21 & 109 \\
\hline 100 & 0.1 & - & - & - & - \\
\hline 100 & 1 & 0.28 & 94 & 0.18 & 148 \\
\hline 100 & 10 & 0.29 & 92 & 0.26 & 102 \\
\hline
\end{tabular}

Note.

a Turnoff is when the mass fraction $X_{\mathrm{La}}$ or $X_{\mathrm{Ac}}$ drops below $10^{-3}$. The columns show the maximum $Y_{\mathrm{e}}$ and corresponding minimum $\bar{A}_{\text {fin }}$ for which $X_{i} \geqslant 10^{-3}$. A dash (-) denotes that $X_{i}<10^{-3}$ for all $Y_{\mathrm{e}}$, which means there is a neutronrich freeze-out.

where $Y_{\text {seed }}\left(t=t_{n}\right)$ is the abundance of all seed nuclides $(A \geqslant$ 12) at the time that neutrons are exhausted (when $X_{\mathrm{n}} \leqslant 10^{-4}$ ) and $Y_{\text {seed }}(t=0)$ is the initial abundance of seed nuclei. This estimate for the number of fission cycles rests on the assumption that only fission can create additional seed nuclides. When a neutron captures on a seed nuclide, it creates a heavier nuclide, but it will not increase the total number (and hence abundance) of seed nuclides in the ejecta. However, if a heavy nuclide (which is counted as a seed nuclide) fissions, then there are two seed nuclides in its place. Thus comparing the number of heavy nuclides at the time when neutron capture ceases to the initial number of heavy nuclides tells us how many additional heavy nuclides were produced. For example, if $Y_{\text {seed }}\left(t=t_{n}\right)=Y_{\text {seed }}(t=0)$, then no additional heavy nuclides were produced and thus there was no fission cycling, hence $N_{\mathrm{f}}=0$. But if $Y_{\text {seed }}\left(t=t_{n}\right)=3 Y_{\text {seed }}(t=0)$, for example, then (on average) each initial heavy nuclide produced two additional heavy nuclides and so there were two fission cycles, hence $N_{\mathrm{f}}=2$. Note that this method of estimating the number of fission cycles breaks down if nuclides with $A \geqslant 12$ are produced from nuclides with $A<12$, e.g., ${ }^{12} \mathrm{C}$ from three ${ }^{4} \mathrm{He}$.
This happens most prominently at $Y_{\mathrm{e}}$ close to 0.5 and at high entropies, where fission will clearly not occur.

As expected, there are many fission cycles at low $Y_{\mathrm{e}}$ where large amounts of lanthanides and actinides are produced. In the regions with significant fission cycling, $X_{\mathrm{La}}, X_{\mathrm{Ac}}$, and $\epsilon$ are fairly insensitive to $Y_{\mathrm{e}}$ because fission cycling effectively limits the maximum mass of nuclides that are produced to $A \sim 300$. As the ejecta becomes less neutron-rich, fewer fission cycles occur because there are not enough free neutrons to produce fissionable material with $A \gtrsim 250$.

In most panels in Figure 3 we see that the production of actinides is closely tied to fission cycling; actinides go away just after fission cycling stops. If the r-process cannot get to $A \sim 250$, it cannot create actinides and it cannot create fissionable material. Furthermore, in most panels, but especially in $s 1 \tau 1$ and $s 1 \tau 10$ there is an increase in $X_{\mathrm{Ac}}$ and decrease in $X_{\mathrm{La}}$ at the electron fraction where fission cycling stops and just before actinides are not produced. Just as fission cycling stops, the r-process can get to about $A=250$, but not much above. This means that actinides can still be produced, but they are not being fissioned (because only lighter actinides are produced or there are no more free neutrons to initiate fission). Lanthanides have a mass around 150 and so they can be created from fission products. When fission is just turning off, we lose a small source of lanthanides leading to the (small) decline in $X_{\mathrm{La}}$ that can be prominently seen in $s 1 \tau 10$ in Figure 3 at $Y_{\mathrm{e}}=0.17$.

\subsection{Lanthanide Production and Heating Rate in the Full Parameter Space}

Since the amount of lanthanides determines the opacity of the ejecta and the nuclear heating rate determines the amount of energy available for the electromagnetic transient, we are especially interested in how these two quantities are correlated in our parameter space. Figures 4-6 show slices of the final lanthanide and actinide mass fractions, $X_{\mathrm{La}+\mathrm{Ac}}$, and heating rates at 1 day for the extreme and intermediate values of $Y_{\mathrm{e}}, s$, and $\tau$. All the other slice plots are available at http:// stellarcollapse.org/lippunerroberts2015. In the following, the term "lanthanide" will stand for both lanthanides and actinides, unless actinides are specifically mentioned. Unsurprisingly, $X_{\mathrm{La}}$ +Ac depends most strongly on $Y_{\mathrm{e}}$ and the ejecta is lanthanidefree for $Y_{\mathrm{e}} \gtrsim 0.26$. However, even for a very low $Y_{\mathrm{e}}$ of 0.01 , there are some combinations of $s$ and $\tau$ that yield a lanthanidefree ejecta (see upper left panel of Figure 4). Specifically, at high entropies $\left(s \gtrsim 20 k_{\mathrm{B}}\right.$ baryon $\left.^{-1}\right)$ and small expansion timescales $(\tau \lesssim 1 \mathrm{~ms})$, no lanthanides are produced. The reason for this is that neutron capture begins at a lower density because of the high entropy (for a fixed temperature at which neutron capture begins) and therefore the neutron capture timescale is increased. This-in combination with light seed nuclei, a large initial neutron abundance, a potentially $\alpha$-rich freeze-out, and a short dynamical timescale — prevents production of lanthanides and sometimes results in a neutron-rich freeze-out. At lower entropies, the seed nuclei are heavier and the density is higher during the neutron capture period, allowing neutrons to capture on them even at small expansion timescales. And at larger expansion timescales, there is more time for the neutrons to capture on the light seed nuclei even at very high entropies. This is reflected in the upper right panel of Figure 5 where no lanthanides are produced at small expansion timescales at $s=100 k_{\mathrm{B}}$ baryon $^{-1}$, and in the upper left panel 


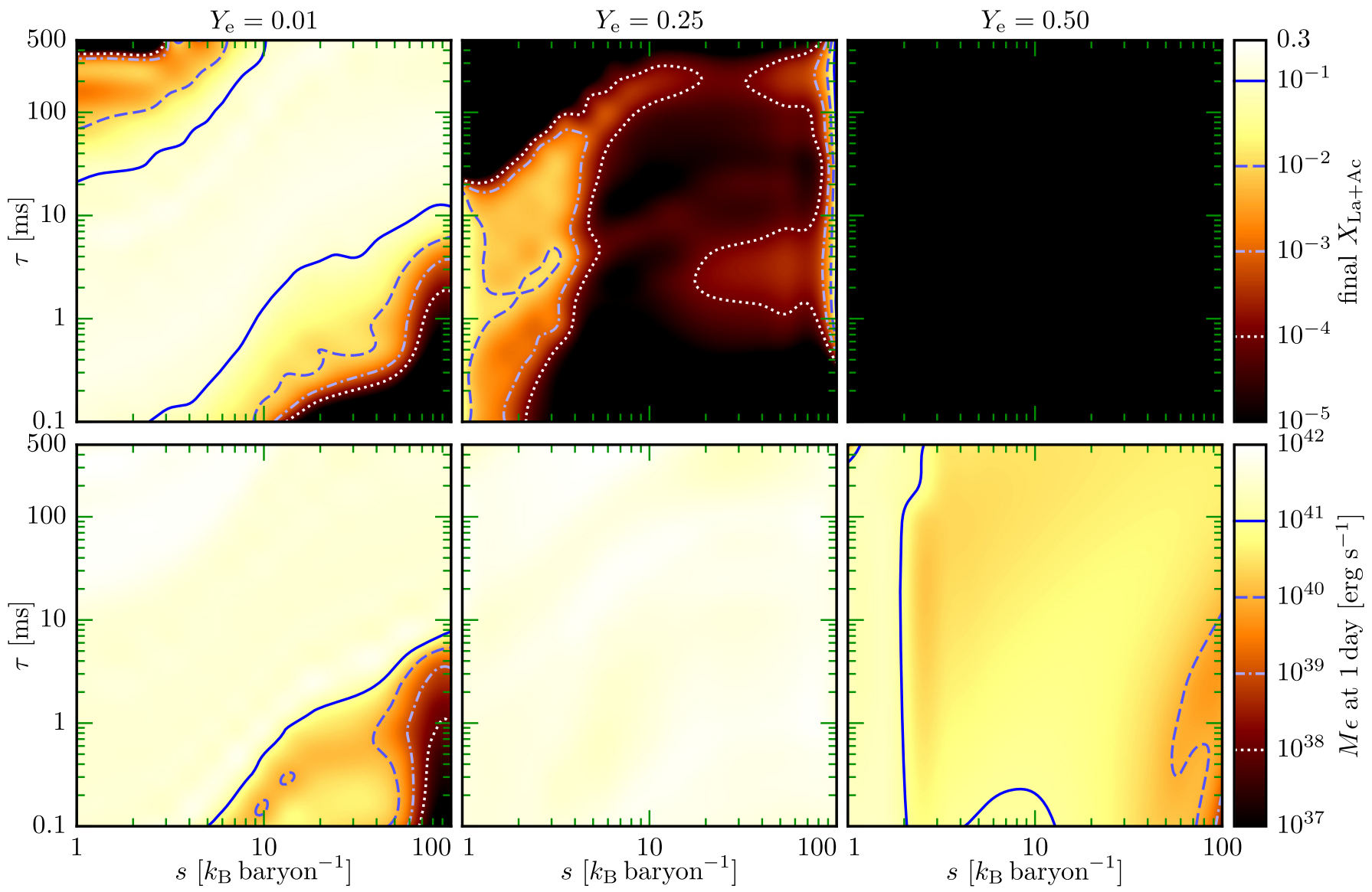

Figure 4. Slices of constant electron fraction showing the lanthanide and actinide mass fraction $X_{\mathrm{La}+\mathrm{Ac}}$ and the heating rate $M \epsilon$ at 1 day with $M=10^{-2} M_{\odot}$. For $Y_{\mathrm{e}}=0.01$, the high-s/small- $\tau$ corner is lanthanide-free because the high entropy produces very light seed nuclides, fewer seed nuclei are produced due to an $\alpha$-rich freeze-out, and neutron capture begins at low density due to the high entropy (see the text for more discussion). The low- $s /$ large- $\tau$ corner is lanthanide-free because the slow expansion timescale results in significant late-time heating, which drives the ejecta back to NSE, but at those late times, $\beta$-decays have significantly raised the electron fraction and so the r-process starts again but at a much higher $Y_{\mathrm{e}}$, which does not produce lanthanides. The $Y_{\mathrm{e}}=0.25$ slice is the transition between lanthanide-rich and lanthanide-free. At low entropies we can still make significant amounts of lanthanides because the seed nuclides are heavy, and at very high entropies we initially have a lot of free neutrons and $\alpha$ particles, which can produce significant amounts of heavy elements. Finally, at $Y_{\mathrm{e}}=0.50$ the material is simply not neutron-rich enough to make any lanthanides. The heating rate at 1 day is quite insensitive to $s$ and $\tau$, except at low $Y_{\mathrm{e}}$, where it is significantly smaller at high entropies and fast expansion timescales because a neutron-rich freeze-out happens. The uniformity in the heating rate is due to the fact that there is an ensemble of nuclides contributing to the heating. And since we are considering the heating at 1 day, we tend to pick up nuclides with similar decay energies (because the decay energy is correlated with the half-life), leading to similar heating rates even if the composition varies.

of Figure 6 where no lanthanides are produced at high entropies at $\tau=0.1 \mathrm{~ms}$.

There is another lanthanide-free corner in the upper left panel of Figure 4 at very large expansion timescales $(\tau \gtrsim 400 \mathrm{~ms})$ and low entropies $\left(s \lesssim 3 k_{\mathrm{B}}\right.$ baryon $\left.^{-1}\right)$. Here, the full r-process is being made, since the material is very neutron-rich, but because the expansion timescale is so long, the density is still quite high (about $10^{10} \mathrm{~g} \mathrm{~cm}^{-3}$ ) when neutron burning ceases. All the heavy elements then decay and considerably heat up the material (to above $7 \mathrm{GK}$ ), which destroys all heavy nuclides via photodissociation and brings the composition back to NSE. Only after tens of seconds has the material cooled down enough for neutron captures to happen again, but by then, $\beta$-decays have raised $Y_{\mathrm{e}}$ to about 0.3 . Thus we now get an r-process with an initial $Y_{\mathrm{e}}$ of 0.3 , which is not neutron-rich enough to produce lanthanides. At faster expansion rates (smaller $\tau$ ) the density falls off faster, resulting in less dramatic heating that cannot force the composition into NSE. Because we obtain the initial density from solving for NSE at the prescribed entropy, $Y_{\mathrm{e}}$, and $T=6 \mathrm{GK}$, the initial density is lower at higher entropies $\left(s \gtrsim 3 k_{\mathrm{B}}\right.$ baryon $\left.^{-1}\right)$ and so even though the density remains close to the initial value at $\tau=500 \mathrm{~ms}$, the density is not high enough to produce heating that results in NSE. This is reflected in the upper left panel of Figure 5 where the ejecta is lanthanide-free at large expansion timescales at $s=1 k_{\mathrm{B}}$ baryon $^{-1}$, and in the upper right panel of Figure 6 where no lanthanides are produced at low entropies at $\tau=500 \mathrm{~ms}$.

The $Y_{\mathrm{e}}=0.25$ slice in Figure 4 is right at the transition from lanthanide-rich to lanthanide-free ejecta. The upper panels of Figures 5 and 6 show clearly that this transition is very sharp at $Y_{\mathrm{e}} \sim 0.22-0.30$. In the upper middle panel of Figure 4 , the low- $s /$ large- $\tau$ corner that is lanthanide-free has expanded and so has the high-s/small- $\tau$ corner, relative to the $Y_{\mathrm{e}}=0.01$ panel. Additionally, lanthanide production is suppressed at intermediate entropies $\left(5 k_{\mathrm{B}}\right.$ baryon $^{-1} \lesssim s \lesssim 90 k_{\mathrm{B}}$ baryon $\left.^{-1}\right)$. At low entropies, we still get an r-process because the seed nuclei are very heavy and thus require fewer neutrons to capture on them to make the r-process distribution. At very high entropies, the initial composition includes a large fraction of free neutrons and $\alpha$ particles. At high entropies, production of seed nuclei via neutron catalyzed triple- $\alpha$ is suppressed 

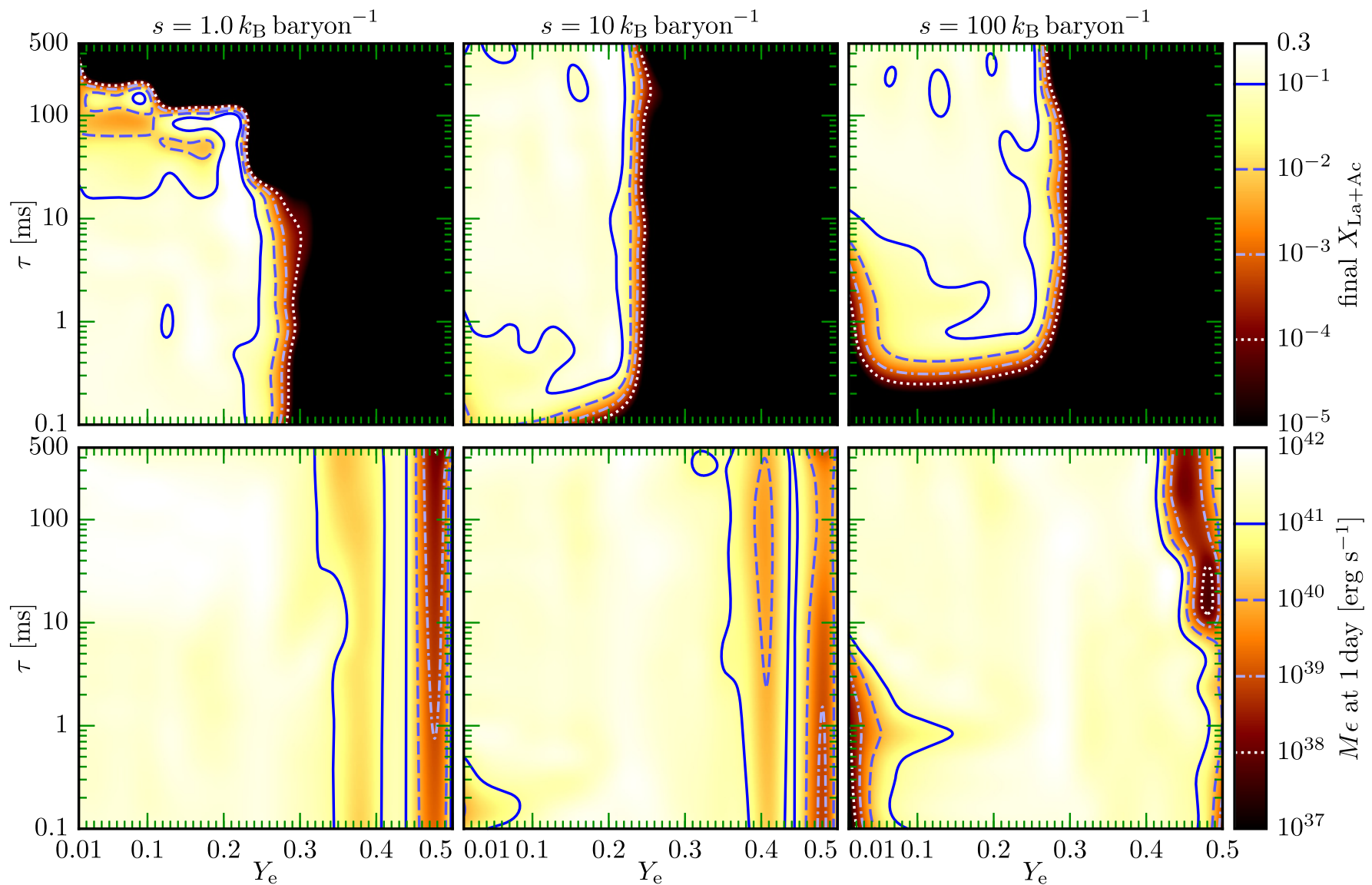

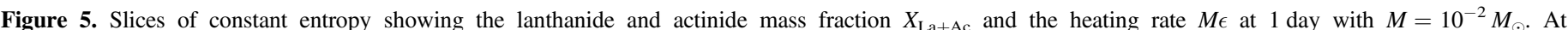

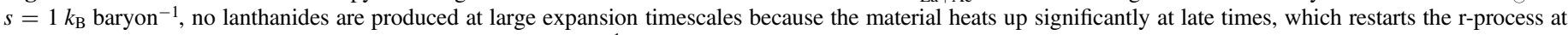

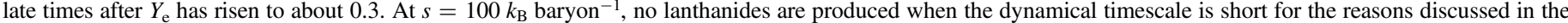

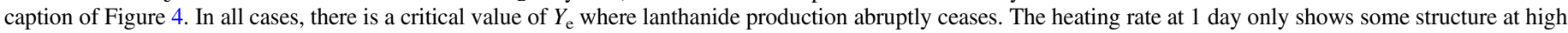

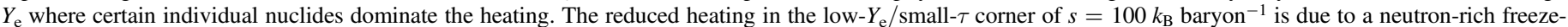
out that occurs there.

(Hoffman et al. 1997), which reduces the number of seed nuclei and thereby increases the neutron-to-seed ratio. These conditions allow for the production of the r-process nuclei. With $Y_{\mathrm{e}} \gtrsim 0.3$, lanthanides are not produced at any entropy and expansion timescale combination, since the ejecta is not neutron-rich enough. In Section 2.2 we discussed in detail how the final lanthanide and actinide mass fractions depend on $Y_{\mathrm{e}}$.

The lower row of panels in Figures 4-6 shows the heating rate (actually $M \epsilon$ where $M=10^{-2} M_{\odot}$ ) at 1 day. For $0.04 \lesssim Y_{\mathrm{e}}$ $\lesssim 0.35$ all the $Y_{\mathrm{e}}$ slices are very similar to the lower middle panel of Figure 4, with virtually no structure. At $Y_{\mathrm{e}}=0.01$, the high- $s$ /small- $\tau$ corner has significantly less heating because the initial density is very low $\left(\rho_{0} \sim 8 \times 10^{5} \mathrm{~g} \mathrm{~cm}^{-3}\right)$ and this, coupled with the rapid expansion timescale $(\tau=0.1 \mathrm{~ms})$ and the fact that the initial composition contains few seed nuclei (98\% of the mass is neutrons), means there is little opportunity for neutron capture. For larger expansion timescales, the initial conditions remain the same (low initial density and $98 \%$ of the mass is neutrons), but because the density decreases more slowly, there is sufficient time for neutrons to capture on the few seed nuclei available and make a full r-process. At lower initial entropies, the initial density is larger (e.g., $4 \times 10^{6} \mathrm{~g} \mathrm{~cm}^{-3}$ at $s=32 k_{\mathrm{B}}$ baryon $^{-1}$ ) so that the density remains higher even with a rapid expansion, giving the neutrons a better chance to capture on seed nuclei-of which there are slightly more available-leading to a moderate r-process. This is reflected in the low $-Y_{\mathrm{e}} /$ small- $\tau$ corner of the lower right panel in Figure 5 and in the low $-Y_{\mathrm{e}} /$ high- $S$ corner of the lower left panel in Figure 6.

For $Y_{\mathrm{e}} \gtrsim 0.35$ we start to see large variations in the heating rate at 1 day as a function of $Y_{\mathrm{e}}$, which can be seen in all lower panels in Figures 5 and 6 . But the heating is still quite insensitive to $s$ and $\tau$, as the lower right panel of Figure 4 shows. This variation as a function of $Y_{\mathrm{e}}$ at high $Y_{\mathrm{e}}$ can also be seen in Figure 3. There is a pronounced peak in the heating rate at 1 day at $Y_{\mathrm{e}}=0.425$ in all but the $s=100 k_{\mathrm{B}}$ baryon $^{-1}$ cases. This peak is due to the decay of ${ }^{66} \mathrm{Cu}$ (half-life of 5 minutes) which comes from the decay of ${ }^{66} \mathrm{Ni}$, which has a half-life of $55 \mathrm{hr} .{ }^{66} \mathrm{Ni}$ has 28 protons and 38 neutrons and so its electron fraction is $28 / 66 \approx 0.424$, which is very close to $Y_{\mathrm{e}}=0.425$, the initial electron fraction of the material. Thus the initial NSE distribution contains a larger quantity of ${ }^{66} \mathrm{Ni}$ at $Y_{\mathrm{e}}=0.425$ than at different $Y_{\mathrm{e}}$, which leads to excessive heating via the decay chain described above because ${ }^{66} \mathrm{Cu}$ has a fairly large $Q$ value of $2.6 \mathrm{MeV}$. At $s=100 k_{\mathrm{B}}$ baryon $^{-1}$ the initial neutronto-seed ratio is much larger than at lower entropies and so significant neutron burning occurs even at high $Y_{\mathrm{e}}$, which washes out the strong dependence of the heating rate at 1 day on $Y_{\mathrm{e}}$. 

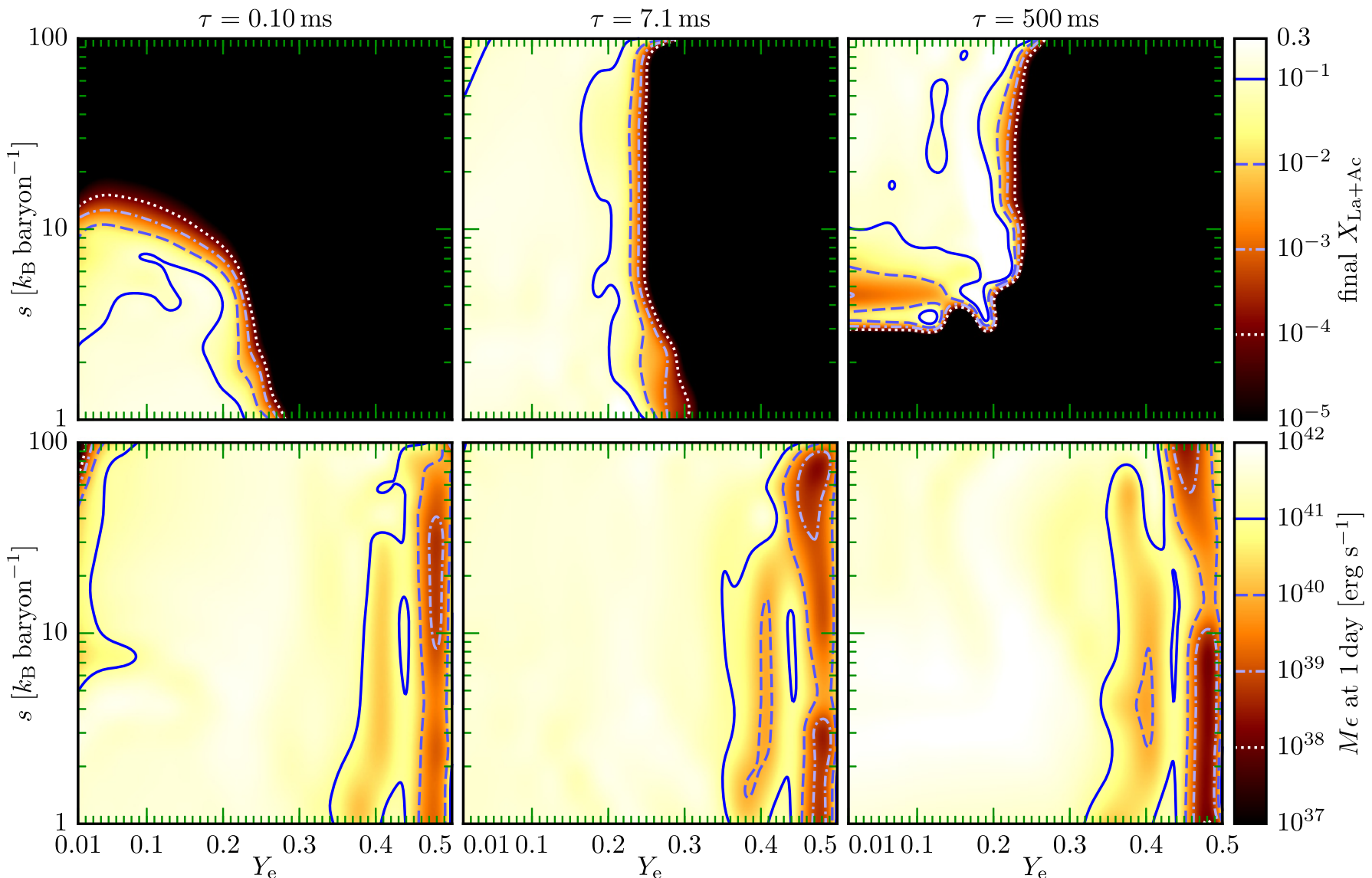

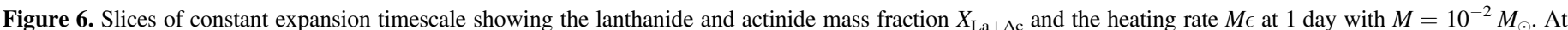



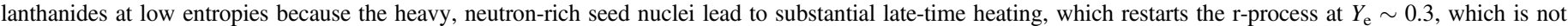

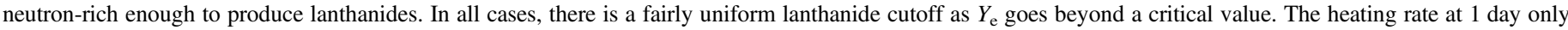
shows structure at high $Y_{\mathrm{e}}$ where certain individual nuclides dominate the heating.

In Figure 3, there are also large minima in the heating rate at 1 day in all but the $s=100 k_{\mathrm{B}}$ baryon $^{-1}$ cases at electron fractions between 0.45 and 0.48 , depending on $s$ and $\tau$. These minima can also be seen in Figures 5 and 6. In those cases, NSE preferentially produces stable isotopes in the initial composition, which drastically reduces the heating. For example, the cases with $s=1 k_{\mathrm{B}}$ baryon $^{-1}$ have the minima at $Y_{\mathrm{e}}=0.465$ and over $80 \%$ of the initial mass is either stable or has a half-life of more than 100 days. The most abundant nuclide (37\% of the mass) is ${ }^{56} \mathrm{Fe}$, which is stable and has $Y_{\mathrm{e}}=26 / 56 \approx 0.464$, which is why the minimum occurs at $Y_{\mathrm{e}}=0.465$, because that favors ${ }^{56} \mathrm{Fe}$ the most. As another example, the $s=10 k_{\mathrm{B}}$ baryon $^{-1}$ cases have the minima at $Y_{\mathrm{e}}=0.45$, where ${ }^{58} \mathrm{Fe}$ and ${ }^{62} \mathrm{Ni}$ are preferentially produced by NSE, which have electron fractions of 0.448 and 0.452 , respectively.

As in Section 2.2, we do not find a significant correlation between the amount of lanthanides and actinides produced with the heating rate at 1 day. The heating rate at 1 day is very uniform at values of $Y_{\mathrm{e}}$ where lanthanides are produced. Since we are looking at the heating rate at a specific time, we will always pick out the nuclides with a half-life of about 1 day (or decay products of nuclides that decay on a one-day timescale). Because the decay energy is correlated with the half-life and because we always have a collection of different nuclides, we obtain roughly the same heating rate at 1 day regardless of the exact composition of the ejecta. This is no longer true at higher $Y_{\mathrm{e}}$, where the composition can be dominated by individual nuclides, which then determine the heating rate.

\subsection{Fitted Nuclear Heating Rates}

For each nucleosynthesis calculation, we calculate a parametric fit for the nuclear heating rate $\epsilon(t)$ between 0.1 and 100 days (the fit window). The fit has the form

$$
\hat{\epsilon}(t)=A t^{-\alpha}+B_{1} e^{-t / \beta_{1}}+B_{2} e^{-t / \beta_{2}}+B_{3} e^{-t / \beta_{3}},
$$

where $t$ and $\beta_{i}$ are in days and $\hat{\epsilon}(t)$ is in $\operatorname{erg~s}^{-1} \mathrm{~g}^{-1}$. We use at most six parameters for the fit, so either $A$ and $\alpha$ are zero or one or more of $B_{i}$ and $\beta_{i}$ are zero. We use a weighted fit where the range 0.1-100 days has a weight of one and the weight decreases linearly to zero in logspace from 0.1 to 0.05 days and from 100 to 200 days. We use a heuristic method to find the global best fit for all six types of fits (power law with 0,1 , or 2 exponentials, or 1,2, or 3 exponentials without a power law term). The best of these six fits is then selected with a small penalty term for the number of parameter pairs. The fitting error is multiplied by 1.1 for each parameter pair in excess of one, so that we do not pick up meaningless parameters that improve the fit by less than $10 \%$.

For consistency, we calculate the fitting error at the same times $t_{i}$ for all cases and we interpolate the actual heating rate to 


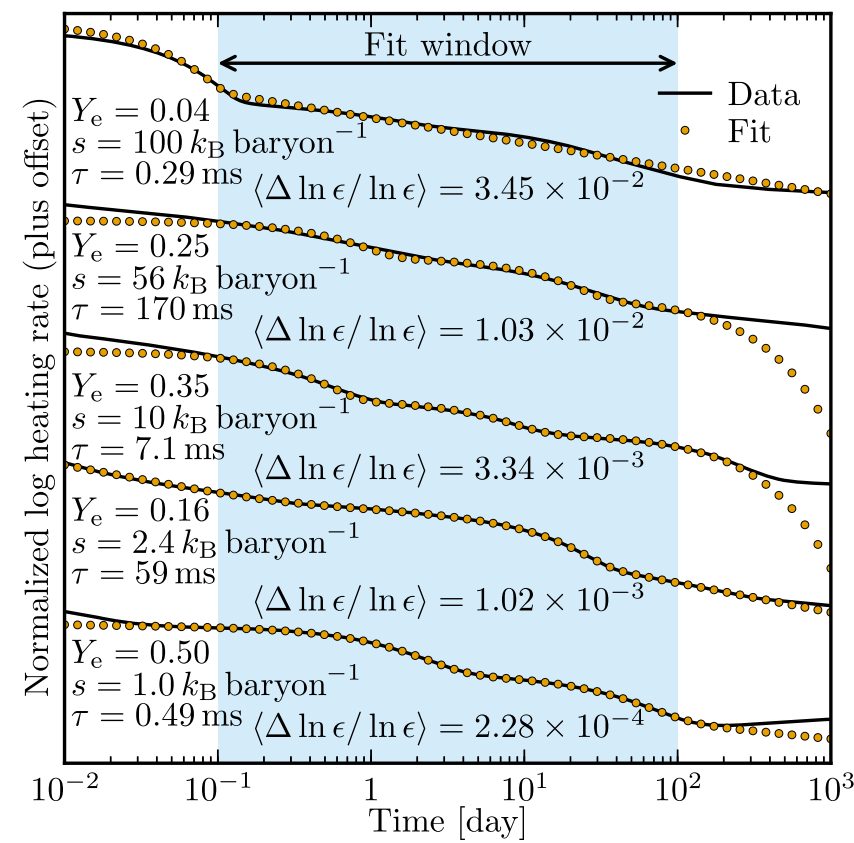

Figure 7. Some heating rate fits showing the fits with the largest and smallest error, and fits with errors in between. The heating rate is only fitted inside the fit window (0.1-100 days). We use a power law with up to two exponential terms, or up to three exponential terms without a power law show in Equation (4), whichever produces the best fit. The fit error $\langle\Delta \ln \epsilon / \ln \epsilon\rangle$ is defined in Equation (6). As the second and third case from the top show, the fit can be quite bad outside the fit window. This is no surprise since we do not fit the data outside the fit window and because we only use up to three exponential terms. In reality, there are hundreds of individual nuclides contributing to the total heating rate and each one contributes a different exponential term.

those times, which are 500 points uniformly sampled in logspace between $10^{-2}$ and $10^{3}$ days (however, points before 0.05 days and after 200 days have zero weight and thus do not contribute to the fitting error, as explained above). The fit error used for finding the optimal fit parameters is the sum of squares of the $\log$ difference, i.e.,

$$
\text { fit error }=\sum_{i} w_{i}\left(\ln \epsilon\left(t_{i}\right)-\ln \hat{\epsilon}\left(t_{i}\right)\right)^{2},
$$

where $w_{i}$ is the weight of time $t_{i}$. This error measure works well for the optimization algorithm to find the best parameters, but it carries little physical meaning. To be able to intuitively judge the quality of a particular fit, we define the mean fractional log error as

$$
\left\langle\frac{\Delta \ln \epsilon}{\ln \epsilon}\right\rangle=\left\langle\frac{\left|\ln \epsilon\left(t_{i}\right)-\ln \hat{\epsilon}\left(t_{i}\right)\right|}{\ln \epsilon\left(t_{i}\right)}\right\rangle,
$$

where the average runs over all times $t_{i}$ such that 0.1 days $\leqslant t_{i} \leqslant 100$ days. We only fit the total heating rate, but we also provide the average heating contribution due to fission reactions in the fit window.

The best and worst heating rate fits, as well as some fits of intermediate quality, are shown in Figure 7 . About $80 \%$ of all high-resolution sym0 fits have $\langle\Delta \ln \epsilon / \ln \epsilon\rangle \leqslant 0.5 \%$ and about $95 \%$ have a mean fractional $\log$ error of at most $1 \%$. Since we do not include $\beta$-delayed fission reactions, the heating due to fission in our fit window (0.1-100 days) is solely due to spontaneous fission and it is close to constant during the fit window because there is usually one nuclide that dominates the fission heating. In $85 \%$ of all cases it varies by less than a factor of two within the fit window, and in $99 \%$ of all cases it varies by less than a factor of three. Thus it is sufficient to report the geometric mean of the heating rate due to fission over the fit window. Fits to the heating rates over our entire parameter space are available at http://stellarcollapse.org/ lippunerroberts 2015 .

\subsection{Dominant Nuclear Decays}

To determine the particular nuclei that are likely to power kilonovae, we integrate the fractional heating contributions of all nuclides to find out which nuclides contribute most to the heating. For a single nucleosynthesis calculation, we know the total heating rate $\epsilon(t)$ as a function of time and we can calculate the heating rate $\epsilon_{i}(t)$ due to nuclide $i$ as a function of time. $\epsilon_{i}(t)$ is calculated as

$$
\epsilon_{i}(t)=N_{\mathrm{A}} \sum_{\alpha \in \mathcal{D}_{i}} \lambda_{\alpha}(t) Q_{\alpha} Y_{i}(t),
$$

where $\alpha$ is an index of a reaction in the reaction network and it runs over the set $\mathcal{D}_{i}$, which is the set of all reactions that destroy exactly one nuclide $i$. $N_{\mathrm{A}}$ is the Avogadro constant in baryon $\mathrm{g}^{-1}, \lambda_{\alpha}(t)$ is the reaction rate of reaction $\alpha$ in $\mathrm{s}^{-1}, Q_{\alpha}$ is the energy released in reaction $\alpha$ in erg, and $Y_{i}(t)$ is the number abundance of nuclide $i$ in baryon ${ }^{-1}$. Note that the total heating rate is $\epsilon(t)=\sum_{i} \epsilon_{i}(t)$, where $i$ runs over all nuclear species in the network.

At any given time $t$, we can now calculate the fractional heating contribution of nuclide $i$ as $\epsilon_{i}(t) / \epsilon(t)$, which is the fraction of the total heating rate at time $t$ that is solely due to the decay of nuclide $i$. These fractional heating contributions tell us which nuclides dominate the heating at a given time. To quantify which nuclides dominate the heating over a period of time, we define the integrated fractional heating contribution $f_{i}$ as

$$
f_{i}=\frac{1}{\ln t_{1} / t_{0}} \int_{t_{0}}^{t_{1}} \frac{\epsilon_{i}(t)}{\epsilon(t)} d \ln t
$$

where $t_{0}=0.1$ days and $t_{1}=100$ days are the beginning and end of our heating rate fit window. We integrate in logspace to equally weigh contributions at early and late times. Since we know $\epsilon_{i}$ and $\epsilon$ only at specific time steps $t_{k}$, we approximate the integral as

$$
f_{i} \sim \frac{1}{\ln t_{1} / t_{0}} \sum_{t_{0} \leqslant t_{k} \leqslant t_{1}} \frac{\epsilon_{i}\left(t_{k}\right)}{\epsilon\left(t_{k}\right)} \ln \frac{t_{k+1}}{t_{k}} .
$$

If no $t_{k}$ is equal to $t_{0}$ or $t_{1}$, we add these two endpoints to the sum and interpolate $\epsilon_{i}$ and $\epsilon$ at those points.

Note that we calculate $f_{i}$ for each nuclide $i$ in a single nucleosynthesis calculation. So we should really say $f_{i}\left(Y_{\mathrm{e}}, s, \tau\right)$, because $f_{i}$ will be different for the same nuclide $i$ in different nucleosynthesis calculations since different amounts of nuclide $i$ are be produced, depending on $Y_{\mathrm{e}}, s$, and $\tau$. To get an idea of which nuclides have the biggest influence on the heating rate over a range of $Y_{\mathrm{e}}, s$, and $\tau$, we average $f_{i}$ over multiple nucleosynthesis calculations in our parameter space. We call this the average integrated fractional 
Table 3

Average Integrated Fractional Heating Contributions $\bar{f}_{i}$ of the High-resolution sym $0^{\text {a }}$ Runs

\begin{tabular}{|c|c|c|c|c|c|c|c|c|c|}
\hline \multicolumn{8}{|c|}{$Y_{\mathrm{e}}$ Bins $^{\mathrm{b}}$} & \multirow{2}{*}{\multicolumn{2}{|c|}{$\frac{\text { Overall }^{\mathrm{c}}}{\left(0<Y_{\mathrm{e}} \leqslant 0.5\right)}$}} \\
\hline \multicolumn{2}{|c|}{$0<Y_{\mathrm{e}} \leqslant 0.125$} & \multicolumn{2}{|c|}{$0.125<Y_{\mathrm{e}} \leqslant 0.250$} & \multicolumn{2}{|c|}{$0.250<Y_{\mathrm{e}} \leqslant 0.375$} & \multicolumn{2}{|c|}{$0.375<Y_{\mathrm{e}} \leqslant 0.5$} & & \\
\hline Nuclide & $\bar{f}_{i}$ & Nuclide & $\bar{f}_{i}$ & Nuclide & $\bar{f}_{i}$ & Nuclide & $\bar{f}_{i}$ & Nuclide & $\bar{f}_{i}$ \\
\hline${ }^{200} \mathrm{Au}$ & $4.46 \%$ & ${ }^{131} \mathrm{I}$ & $5.52 \%$ & ${ }^{72} \mathrm{Ga}$ & $5.91 \%$ & ${ }^{57} \mathrm{Ni}$ & $10.83 \%$ & ${ }^{66} \mathrm{Cu}$ & $4.42 \%$ \\
\hline${ }^{128} \mathrm{Sb}$ & $4.26 \%$ & ${ }^{128} \mathrm{Sb}$ & $4.66 \%$ & ${ }^{132} \mathrm{I}$ & $5.00 \%$ & ${ }^{59} \mathrm{Fe}$ & $7.47 \%$ & ${ }^{89} \mathrm{Sr}$ & $3.51 \%$ \\
\hline${ }^{249} \mathrm{Bk}$ & $4.23 \%$ & ${ }^{132} \mathrm{Te}$ & $3.78 \%$ & ${ }^{59} \mathrm{Fe}$ & $4.77 \%$ & ${ }^{89} \mathrm{Sr}$ & $5.21 \%$ & ${ }^{57} \mathrm{Ni}$ & $3.18 \%$ \\
\hline${ }^{252} \mathrm{Cf}$ & $3.09 \%$ & ${ }^{129} \mathrm{Sb}$ & $2.85 \%$ & ${ }^{103} \mathrm{Ru}$ & $3.24 \%$ & ${ }^{61} \mathrm{Cu}$ & $3.20 \%$ & ${ }^{131} \mathrm{I}$ & $2.59 \%$ \\
\hline${ }^{133} \mathrm{I}$ & $3.09 \%$ & ${ }^{127} \mathrm{Sb}$ & $2.79 \%$ & ${ }^{91} \mathrm{Y}$ & $3.08 \%$ & ${ }^{62} \mathrm{Cu}$ & $3.04 \%$ & ${ }^{78} \mathrm{As}$ & $2.27 \%$ \\
\hline${ }^{202} \mathrm{Au}$ & $2.89 \%$ & ${ }^{140} \mathrm{La}$ & $2.56 \%$ & ${ }^{66} \mathrm{Cu}$ & $2.97 \%$ & ${ }^{56} \mathrm{Ni}$ & $3.00 \%$ & ${ }^{72} \mathrm{Ga}$ & $2.05 \%$ \\
\hline${ }^{135} \mathrm{I}$ & $2.65 \%$ & ${ }^{129} \mathrm{Te}$ & $2.25 \%$ & ${ }^{112} \mathrm{Ag}$ & $2.96 \%$ & ${ }^{72} \mathrm{Ga}$ & $2.95 \%$ & ${ }^{77} \mathrm{Ge}$ & $2.02 \%$ \\
\hline
\end{tabular}

Notes.

${ }^{\mathrm{a}}$ Symmetric fission reactions that do not create free neutrons.

${ }^{\mathrm{b}}$ The $\overline{f_{i}}$, s shown in these columns are averaged over all nucleosynthesis calculations (with different initial electron fractions, entropies, and expansion timescales) whose $Y_{\mathrm{e}}$ falls within the $Y_{\mathrm{e}}$ bin.

${ }^{\mathrm{c}}$ The $\bar{f}_{i}$ 's shown in this column are averaged over the entire parameter space.

heating contribution $\bar{f}_{i}$ and calculate it as

$$
\bar{f}_{i}=\frac{1}{|\mathcal{Y}||\mathcal{S}||\mathcal{T}|} \sum_{Y_{\mathrm{e}} \in \mathcal{Y}} \sum_{s \in \mathcal{S} \tau \in \mathcal{T}} \sum_{i}\left(Y_{\mathrm{e}}, s, \tau\right),
$$

where $\mathcal{Y}, \mathcal{S}$, and $\mathcal{T}$ are the sets of values of $Y_{\mathrm{e}}, s$, and $\tau$, respectively, that we are averaging over, and $|\mathcal{Y}|,|\mathcal{S}|$, and $|\mathcal{T}|$ are the cardinalities of those sets, i.e., the number of elements in the sets. Note that this method of averaging is meaningful because we are considering the fractional heating contribution of nuclide $i$ and not the absolute heating contribution, and furthermore, we normalize $f_{i}\left(Y_{\mathrm{e}}, s, \tau\right)$ in the same way for each nucleosynthesis calculation. The final number $\bar{f}_{i}$ that we obtain is a number between 0 and 1 and it tells us that nuclide $i$ is responsible for this fraction of the total heating rate between 0.1 and 100 days averaged over a certain set of parameters $Y_{\mathrm{e}}, s$, and $\tau$. Note that $\bar{f}_{i}$ is not intended to be used to estimate the absolute amount of heating due to nuclide $i$, because the absolute amount of heating can vary greatly between the different nucleosynthesis cases over which we averaged to obtain $\bar{f}_{i}$. Rather, $\bar{f}_{i}$ is intended to quantify how important different nuclides are in the makeup of the total radioactive heating rate over a wide range of possible kilonovae. This can help inform experiments that are measuring the $\beta$-decay properties of nuclides produced in the r-process. To model the r-process and associated kilonovae more accurately, it would be more beneficial to have precise measurements of the $\beta$-decay properties of nuclides that have a larger $\bar{f}_{i}$ than of nuclides with smaller $\bar{f}_{i}$.

Table 3 shows the 10 most dominant heating nuclides and their average integrated fractional heating contributions $\bar{f}_{i}$. The $\bar{f}_{i}$, s are averaged over different high-resolution sym0 (symmetric fission with no free neutrons) runs in different $Y_{\mathrm{e}}$ bins and over the entire range of entropies $\left(1 k_{\mathrm{B}}\right.$ baryon $^{-1} \leqslant s \leqslant 100 k_{\mathrm{B}}$ baryon $\left.^{-1}\right)$ and expansion timescales $(0.1 \mathrm{~ms} \leqslant \tau \leqslant 500 \mathrm{~ms})$. In each $Y_{\mathrm{e}}$ bin, the nuclides are sorted with decreasing $\bar{f}_{i}$. We only look at the $Y_{\mathrm{e}}$-dependence of the dominant heating nuclides because the r-process depends very strongly on $Y_{\mathrm{e}}$, while it is quite insensitive to entropy (e.g.,
Freiburghaus et al. 1999, also see Figure 1). Only the 10 most dominant heating nuclides are shown here, the full table, and the tables of the runs with different fission reactions, are available at http://stellarcollapse.org/lippunerroberts2015. The single most important nuclide for heating between 0.1 and 100 days is ${ }^{132}$ I. It dominates over all other nuclides by a factor of at least 3-10 and it especially dominates at low initial $Y_{\mathrm{e}}{ }^{132} \mathrm{Sn}$ is doubly magic (50 protons and 82 neutrons) and so it gets produced in high quantities in the r-process. Within minutes, ${ }^{132} \mathrm{Sn}$ decays to ${ }^{132} \mathrm{Sb}$ which decays to ${ }^{132} \mathrm{Te} .{ }^{132} \mathrm{Te}$ has a half-life of 3.2 days and so it decays in the middle of our fit window where we are looking at the heating contributions. But the decay of ${ }^{132} \mathrm{Te}$ to ${ }^{132} \mathrm{I}$ has a $Q$-value of only about $500 \mathrm{keV}$, while ${ }^{132} \mathrm{I}$ decays to the stable isotope ${ }^{132} \mathrm{Xe}$ (which is in the middle of the second r-process peak) with a half-life of only $2.3 \mathrm{hr}$ and a $Q$-value of $3.6 \mathrm{MeV}$. Thus we get a large heating contribution from ${ }^{132} \mathrm{I}$.

As is to be expected, at very low $Y_{\mathrm{e}}$ (between 0 and 0.125 ), most of the heating comes from nuclei that form the second $(A \sim 130)$ and third $(A \sim 200)$ r-process peaks. A few very heavy nuclides $(A \sim 250)$ contribute. At higher $Y_{\mathrm{e}}$ (between 0.125 and 0.25 ), the 10 most significantly contributing nuclides are all in the second peak, since anything in the third peak and beyond is more difficult to produce. The nuclides we find to be the dominant source of heating at low initial $Y_{\mathrm{e}}$ are consistent with the dominant $\beta$-decay nuclei that Metzger et al. (2010) found. They only investigated a $Y_{\mathrm{e}}=0.1$ outflow and we confirm that this result holds for a range of electron fractions below 0.25 .

At $Y_{\mathrm{e}}$ between 0.25 and 0.375 there is a mix of significant contributers from the first $(A \sim 88)$ and second peaks. There are also some iron peak elements, but most isotopes on the neutron-rich side of the iron peak have half-lives that are either too short or too long for our fit window. Notable exceptions are ${ }^{59} \mathrm{Fe},{ }^{66} \mathrm{Ni},{ }^{67} \mathrm{Cu}$, and ${ }^{72} \mathrm{Ga}$. We do indeed see significant contributions from ${ }^{72} \mathrm{Ga}$ and ${ }^{59} \mathrm{Fe}$. Instead of ${ }^{66} \mathrm{Ni}$, we see its $\beta$-decay product, ${ }^{66} \mathrm{Cu}$, which has a much larger $Q$-value (2.6 MeV instead of $250 \mathrm{keV}$ ) and a half-life of 5 minutes. ${ }^{67} \mathrm{Cu}$ does not contribute significantly because of its relatively low $Q$-value of $560 \mathrm{keV}$. Finally, at very high $Y_{\mathrm{e}}$ (between 0.375 
and 0.5 ) there are significant significant contributers from the proton-rich side of stability around the iron peak. ${ }^{57} \mathrm{Ni}$ dominates over ${ }^{56} \mathrm{Ni}$ because it has one more neutron-thus it is a bit easier to produce in slightly neutron rich conditions $\left(Y_{\mathrm{e}}<0.5\right)$-and the $\beta^{+}$-decay $Q$-value of ${ }^{57} \mathrm{Ni}$ is a bit larger than that of ${ }^{56} \mathrm{Ni}(3.3 \mathrm{MeV}$ versus $2.1 \mathrm{MeV})$. Both nuclides, however, have a half-life that is right inside our fit window, which is why both contribute significantly to the total heating rate.

The cases that produce significant amounts of actinides also produce nuclides that undergo spontaneous fission. In those cases, the heating due to fission becomes dominant toward the end of the fit window (at about 100 days) but it is subdominant throughout the rest of the fit window. The nuclides that contribute the most to fission induced heating across the entire parameter space are ${ }^{249} \mathrm{Bk},{ }^{252} \mathrm{Cf}$, and ${ }^{241} \mathrm{Pu}$, which have average integrated fractional fission heating contributions of $33 \%, 21 \%$, and $19 \%$, respectively. These numbers are $\bar{f}_{i}$ defined in Equation (10) averaged over the entire parameter space, but the $f_{i}$ 's of the individual nucleosynthesis calculations defined in Equation (8) were calculated using only fission reactions in $\epsilon_{i}(t)$ (cf. Equation (7)) and with $\epsilon(t)$ being the total heating rate due to fission alone. In other words, averaged over all runs in the entire parameter space and averaged in logspace over all times between 0.1 and 100 days, ${ }^{249} \mathrm{Bk}$ accounts for $33 \%$ of the entire heating due to fission, and similarly for the other nuclides. If $\beta$-delayed fission were included in our reaction network, it would likely significantly alter the contribution of fission to the heating rate at low electron fraction. For higher electron fractions, the neglect of betadelayed fission is unlikely to be important since very little fissible material is produced.

\section{LIGHT CURVES}

To test how variations in the late-time nuclear heating rate and composition affect possible electromagnetic transients associated with neutron star mergers, we calculate light curves using a simplified gray radiative transport scheme in a spherically symmetric outflow.

\subsection{Radiative Transfer Methods}

The ejecta is assumed to expand homologously, such that $r=v t$. The density structure of the outflow is then described by

$$
\rho(t, r)=\rho_{0}(r / t)\left(\frac{t}{t_{0}}\right)^{-3} .
$$

SkyNet gives a heating rate $\epsilon(t)$, which is the total amount of energy released per unit mass and per unit time due to nuclear reactions. The majority of this energy is carried away by neutrinos, but some fraction, say $f$, is thermalized in the material. So $f \epsilon(t)$ is the heating rate of the material due to nuclear reactions and decays.

For homologous outflows, the velocity can be taken as a Lagrangian coordinate. Writing down the gray, Lagrangian radiative transport equations to first order in $v / c$ (e.g., Mihalas \& Weibel-Mihalas 1999), using the velocity as the Lagrangian coordinate, and including energy release from nuclear reactions gives

$$
\frac{d E}{d t}+\frac{2 E}{t}+\frac{1}{v^{2} t} \frac{\partial}{\partial v}\left(v^{2} F\right)=\rho c \kappa\left(a T^{4}-E\right)
$$

$$
\begin{gathered}
\frac{d F}{d t}+\frac{1}{t} \frac{\partial}{\partial v}(\mathcal{F} E)+\frac{3 \mathcal{F}-1}{v t} E=-\rho c \kappa F, \\
\frac{d u}{d t}+\frac{3 P}{\rho t}=f \epsilon+c \kappa\left(E-a T^{4}\right),
\end{gathered}
$$

where $E$ is the radiation energy density, $t$ is the time since merger, $v$ is the velocity measured in units of the speed of light $c, F$ is the radiation flux, $\rho$ is the density given in Equation (11), $\kappa$ is the opacity, $a=4 \sigma / c$ is the radiation constant where $\sigma$ is the Stefan-Boltzmann constant, $T$ is the temperature of the fluid, $\mathcal{F}$ is the Eddington factor (i.e., the ratio of the radiation pressure to the radiation energy density), $u$ is the specific internal energy of the fluid, $p$ is the fluid pressure, $f$ is the fraction of the heating rate $\epsilon$ that is thermalized. The heating rate is not entirely thermalized because a large fraction of the nuclear decay energy goes into neutrinos and gamma-rays; neutrinos are lost from the system and gamma-rays are only partially thermalized. To accurately calculate the thermalization fraction, one would need much more detailed information about the $\beta$-decays than what is available in REACLIB and one would also have to do $\gamma$-ray transport. Following Barnes \& Kasen (2013), we adopt $f=0.3$.

The fluid is assumed to be a non-relativistic, non-degenerate ideal gas with molecular weight $\mu$, so that the specific internal energy is $u=3 T /(2 \mu)$. The gray transport equations are discretized in space on a staggered grid, with $E$ and $u$ defined on zone centers and $F$ defined on zone edges. The resulting system of ordinary differential equations is then solved in time using a backward Euler method. Eddington factors are obtained by solving the static Boltzmann transport equation on a tangent ray grid at the beginning of a timestep. This method is similar to the one described in Ensman (1994), specialized to an homologous outflow. The zones are chosen to be logarithmically increasing in size moving away from the maximum radius. This is done to ensure that the radiation decoupling layer is resolved even at high densities.

The density structure is assumed to be described by a broken power law as argued in Chevalier \& Soker (1989). This choice was made mainly to facilitate comparison with Barnes \& Kasen (2013). The power law break and density scale are fixed to give the desired total mass and total kinetic energy of the outflow. We use $M=10^{-2} M_{\odot}$ and $v=0.1 c$, where $c$ is the speed of light, for all light-curve models (e.g., Hotokezaka et al. 2013a; Rosswog 2013; Foucart et al. 2014).

We note that the density evolution in the transport model and the one given in Equation (1) are both proportional to $t^{-3}$, but they have different scale factors. The main point of $\rho(t)$ given in Equation (1) is to control the timescale over which the density changes at the time of nucleosynthesis $(t \lesssim 1 \mathrm{~s})$, but extrapolating this density to late times and assuming that it was the uniform density of a ball of gas expanding with a fixed velocity would lead to superluminal expansion velocities in many cases. Equation (11) gives a much more reasonable estimate of the density at late times after nucleosynthesis is over.

Calculating the exact wavelength and temperature dependent opacity of a mixture is extremely difficult because of the large number of elements and absorption lines involved. Especially the lanthanide and actinide element groups have very complicated line structures and the most sophisticated line structure and opacity calculations have only been done for a 


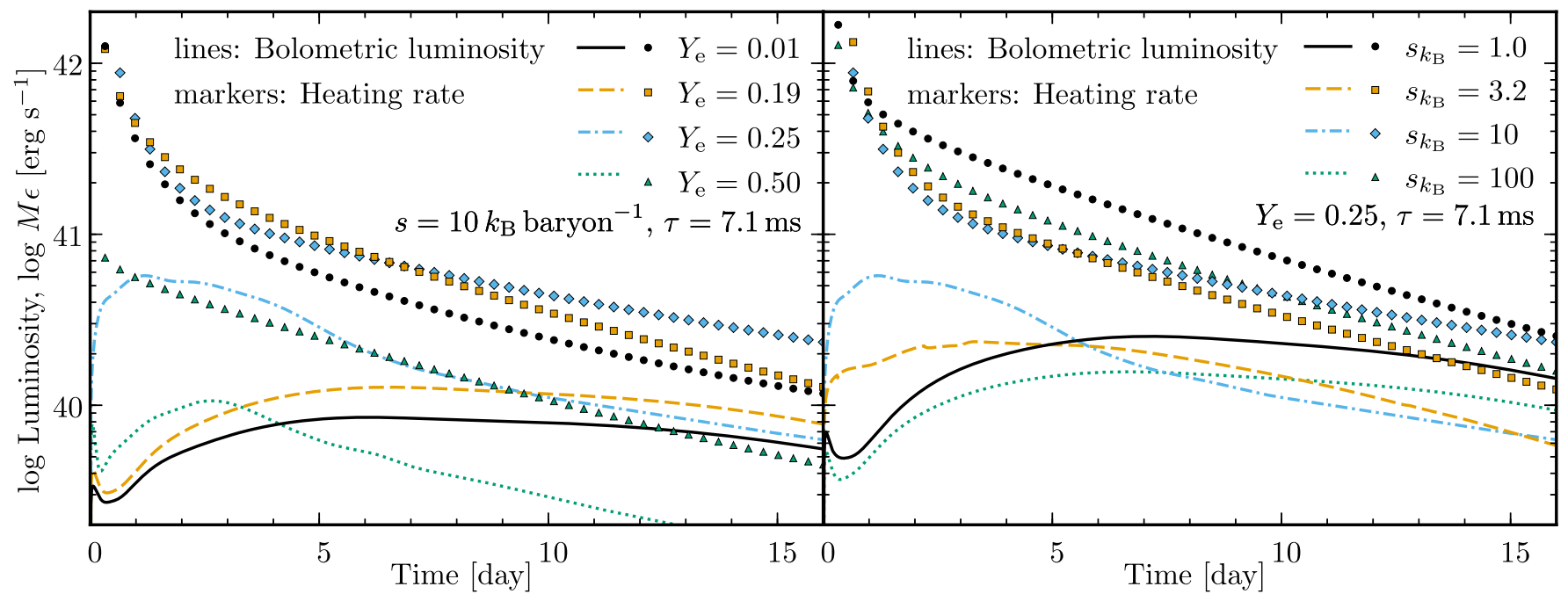

Figure 8. Light curves and heating rates of some selected nucleosynthesis calculations. Left: $Y_{\mathrm{e}}=0.01,0.19,0.25,0.50, s=10 k_{\mathrm{B}}$ baryon ${ }^{-1}$, and $\tau=7.1 \mathrm{~ms}$. With $Y_{\mathrm{e}}=0.01$ and $Y_{\mathrm{e}}=0.19$ we obtain the full r-process and so the ejecta is lanthanide-rich, which drastically increases the opacity, resulting in a dim transient that peaks about a week after the nucleosynthesis event. This is in contrast to the $Y_{\mathrm{e}}=0.25$ case, which has a very similar heating rate as the low- $Y_{\mathrm{e}}$ cases, but does not produce lanthanides, and thus the transient is brighter and peaks earlier. The $Y_{\mathrm{e}}=0.50$ transient is also lanthanide-free and peaks at a few days, but because a significant amount of stable nuclides are produced, the heating is much less, which leads to a dim transient. Right: $Y_{\mathrm{e}}=0.25, s=1.0,3.2,10,100 k_{\mathrm{B}}$ baryon ${ }^{-1}$, and $\tau=7.1 \mathrm{~ms}$. As we saw in Figure 1, the $s=1.0 k_{\mathrm{B}}$ baryon $^{-1}$ and $s=100 k_{\mathrm{B}}$ baryon $^{-1}$ cases are lanthanide-rich, while $s=3.2 k_{\mathrm{B}}$ baryon $^{-1}$ and $s=10 k_{\mathrm{B}}$ baryon $^{-1}$ are lanthanide-free, which is clearly visible in the light curves. Even though $s=3.2 k_{\mathrm{B}}$ baryon $^{-1}$ and $s=10 k_{\mathrm{B}}$ baryon $^{-1}$ have essentially the same heating rate, the $s=3.2 k_{\mathrm{B}}$ baryon $^{-1}$ case is significantly dimmer because it has a small amount of lanthanides. The ejecta of a binary neutron star merger is expected to have entropies between 1 and $10 k_{\mathrm{B}}$ baryon $^{-1}$ (e.g., Goriely et al. 2011; Just et al. 2015).

few representative nuclides (e.g., Kasen et al. 2013). Such detailed opacity calculations are beyond the scope of this work and we use a simple prescription to compute the gray opacity $\kappa$ as a function of temperature $T$ and composition as

$$
\kappa=\kappa_{\mathrm{Fe}}(T)+\sum_{i} \max \left[\kappa_{\mathrm{Nd}}\left(T, X_{i}\right)-\kappa_{\mathrm{Fe}}(T), 0\right],
$$

where $\kappa_{\mathrm{Fe}}(T)$ and $\kappa_{\mathrm{Nd}}\left(T, X_{i}\right)$ are the iron and neodymium opacities given in Kasen et al. (2013). The sum runs over all lanthanide and actinide species with $X_{i}$ being the mass fraction of a particular lanthanide or actinide species. We subtract the iron opacity from the neodymium opacity because $\kappa_{\mathrm{Nd}}\left(T, X_{i}\right)$ given in Kasen et al. (2013) is actually the opacity of a mixture containing $X_{i}$ neodymium and $1-X_{i}$ iron. Our approximation assumes that every lanthanide and actinide contributes the same number of lines with the same distribution in energy. The opacity used in the gray calculation is taken to be the Planck mean opacity, which is appropriate when the wavelength dependent opacity is calculated in the Sobolev approximation (D. Kasen 2015, private communication). At temperatures above $10^{4} \mathrm{~K}$, the opacities are held constant since ionization states which would have been accessed at those temperatures were not included in the original opacity calculation and the opacities there are artificially low (D. Kasen 2015, private communication).

\subsection{Dependence of Kilonova Light Curves on the Outflow Properties}

Figure 8 shows the light curves and heating rates of the cases whose final abundances are shown in Figure 1. In the left panel, the lanthanide-rich cases $\left(Y_{\mathrm{e}}=0.01,0.19\right)$ are about an order of magnitude dimmer than the lanthanide-free case $\left(Y_{\mathrm{e}}=0.25\right)$ and they peak at about a week instead of about a day. The effective temperature at peak of the lanthanide-rich cases is also much lower $(\sim 1600 \mathrm{~K}$ versus $\sim 5700 \mathrm{~K})$ than the temperature of the lanthanide-free case. The heating rates between 0.01 and 100 days, however, are almost identical for those three cases, so the significant differences in the light curves are solely due to the amount of lanthanides present in the ejecta and their effect on the opacity. Comparing the cases $Y_{\mathrm{e}}=0.25$ and $Y_{\mathrm{e}}=0.50$, which are both lanthanide-free, the impact of the heating rate on the light curve can be seen. The heating rate is lower for the $Y_{\mathrm{e}}=0.50$ case, because mostly stable nuclei are produced, leading to less heating. The result is that the light curve of the $Y_{\mathrm{e}}=0.50$ case peaks slightly later (2.6 days versus 1.2 days for $Y_{\mathrm{e}}=0.25$ ), is about an order of magnitude dimmer, and redder (spectral temperature is $\sim 3000 \mathrm{~K}$ compared to $\sim 5700 \mathrm{~K}$ ).

In the left panel of Figure 8, the light curves for $Y_{\mathrm{e}}=0.01$ and $Y_{\mathrm{e}}=0.19$ have a small peak at very early times (about 0.04 days). This early peak comes from our underestimate of the opacity at high temperatures. There is also a small bump at early times in the light curve of the $Y_{\mathrm{e}}=0.50$ case, which is due to the behavior of the heating rate at early times. When determining the actual peak of the light curve, we neglect all peaks earlier than 0.5 days, unless they are more than three times brighter than all peaks after 0.5 days. If there are no peaks after 0.5 days, we pick the brightest peak that is more than three times brighter than the latest peak (which is also before 0.5 days).

The right panel of Figure 8 shows selected light curves with $Y_{\mathrm{e}}=0.25$ and various initial entropies. The cases $s=1 k_{\mathrm{B}}$ baryon $^{-1}$ and $s=100 k_{\mathrm{B}}$ baryon $^{-1}$ produce very typical lanthanide-rich light curves, whereas $s=10 k_{\mathrm{B}}$ baryon $^{-1}$ produces a typical lanthanide-free light curve, and $s=3.2 k_{\mathrm{B}}$ baryon $^{-1}$ produces a light curve that has trace amounts of lanthanides. 


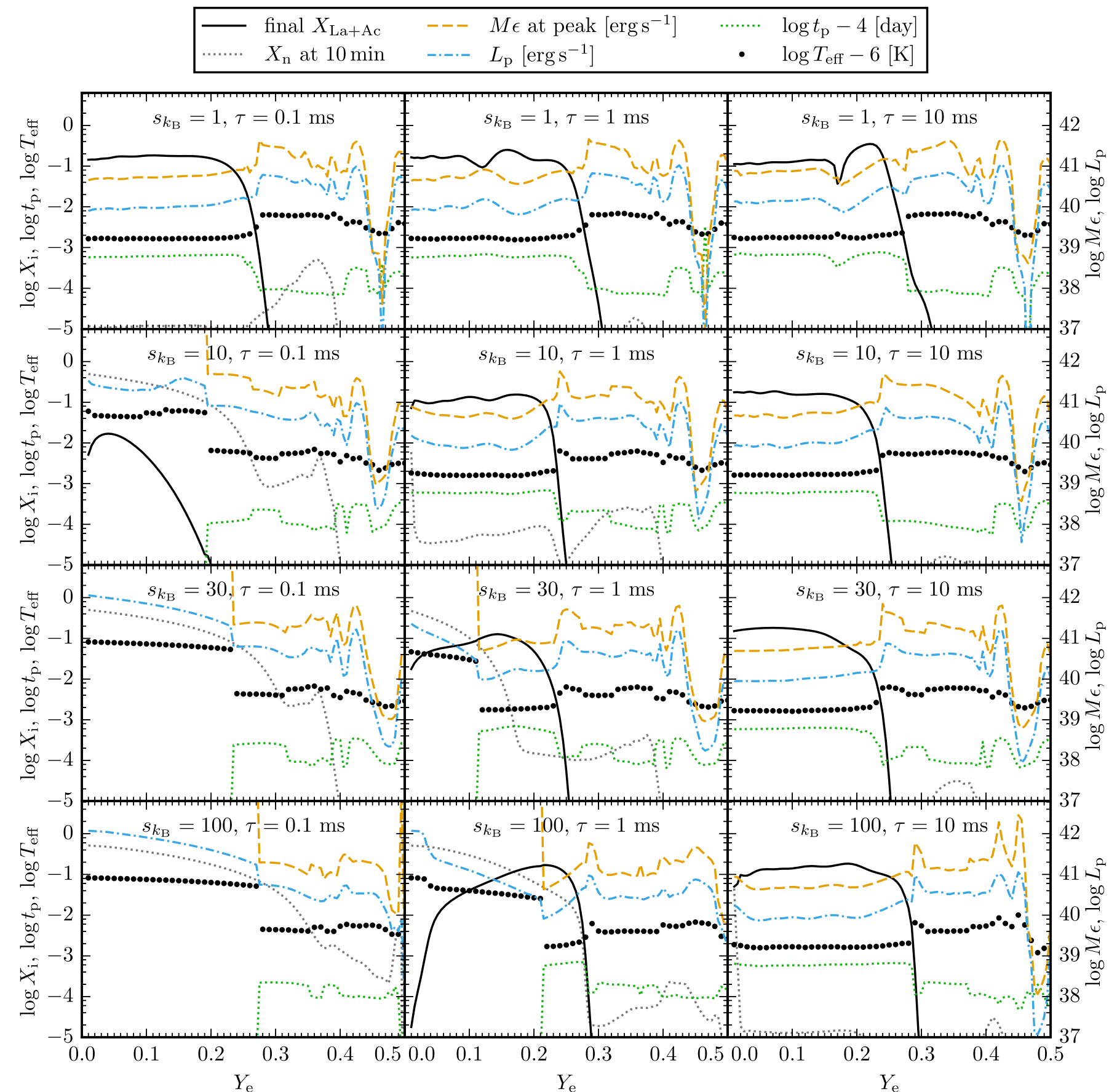

Figure 9. Light-curve results as a function of $Y_{\mathrm{e}}$ for selected values of $s$ and $\tau$. To show how lanthanides and neutron-rich freeze-out impact the light-curve, we again show the lanthanide and actinide abundance $X_{\mathrm{La}+\mathrm{Ac}}$ at peak and the neutron abundance $X_{\mathrm{n}}$ at 10 minutes, which were already shown in Figure 3 . Additionally, we plot the heating rate $M \epsilon$ at peak, with $M=10^{-2} M_{\odot}$, the peak luminosity $L_{\mathrm{p}}$, peak time $t_{\mathrm{p}}$, and the effective blackbody temperature $T_{\text {eff }}$ at peak of the light curve. Note that in the neutron-rich freeze-out cases, the heating rate $M \epsilon$ and the peak timescale $t_{\mathrm{p}}$ go off the scales, their values are $10^{44}-10^{45} \mathrm{erg} \mathrm{s}^{-1}$ and $15-30$ minutes, respectively. As expected, $L_{\mathrm{p}}$ follows the heating rate quite closely, except in the cases where we get a neutron-rich freeze-out. In those cases, we get a bright, very blue transient at early times. The exact point in $Y_{\mathrm{e}}$ of the transition from a neutron-powered transient to an ordinary kilonova in this figure is somewhat arbitrary, since it depends on the exact method of finding the light-curve peak that we choose, as explained in the text. Apart from the neutron-powered transients, the general trend is that we see a slightly dimmer, redder transient at later times if the ejecta is lanthanide-rich, and a brighter, bluer transient at earlier times if it is lanthanide-free. This is consistent with earlier work (e.g., Barnes \& Kasen 2013).

In the cases where we make lanthanides at lower $Y_{\mathrm{e}}$, we expect the peak luminosity to increase and move to earlier times at higher $Y_{\mathrm{e}}$ when the ejecta transitions from lanthaniderich to lanthanide-free, because the large contribution to the opacity from the lanthanides suddenly goes away (Kasen et al. 2013; Tanaka \& Hotokezaka 2013). This is shown in Figure 9.
When lanthanides are not produced, the transient generally becomes brighter, shorter, and bluer. We recall from Figure 3 that the heating rate at 1 day tends to decrease a little when lanthanides go away. Thus the peak luminosity $L_{\mathrm{p}}$ in the lanthanide-free cases is larger not because there is more heating in those cases, but because the peak occurs earlier (due to the 
smaller opacity) and the heating rate is always larger at earlier times than at later times.

Looking at the time $t_{\mathrm{p}}$ of the light curve in Figure 9, we see that the light curve peaks at about 6 days if the ejecta is lanthanide-rich and at about 1 day if the ejecta is lanthanidefree, which is consistent with earlier work (e.g., Roberts et al. 2011; Barnes \& Kasen 2013; Tanaka \& Hotokezaka 2013). At high $Y_{\mathrm{e}}$, where we see some oscillations in the heating rate due to specific nuclides being produced (as explained in Section 2.4), the variation in the heating rate is reflected in the peak luminosity $L_{\mathrm{p}}$ and the peak time $t_{\mathrm{p}}$. More heating results in a brighter transient at later times because the heating keeps the ejecta hotter, and thus the opacity remains high since more excited levels are populated, which increases the number of optically thick lines (Kasen et al. 2013). Conversely, less heating leads to a dimmer transient at earlier times because the ejecta is cooler and thus the opacity is lower. This variation is also reflected in the effective temperature $T_{\text {eff }}$ of the transient, but to a lesser degree. In general, lanthanide-rich transients have $T_{\text {eff }} \sim 1600 \mathrm{~K}$, which peaks at $\lambda \sim 1.8 \mu \mathrm{m}$ in the infrared $\mathrm{H}$ and $\mathrm{K}$ bands. Lanthanide-free transients have $T_{\text {eff }} \sim 6000 \mathrm{~K}$ (although this is a bit lower at very high $Y_{\mathrm{e}}$ where the radioactive heating is reduced), which peaks at $\lambda \sim 480 \mathrm{~nm}$ in the optical B band.

In Figure 9, we can also clearly see that neutron-rich freezeout produces very bright, very early, and very ultraviolet transients. The cleanest examples are $s 30 \tau 0.1$ and $s 100 \tau 0.1$. There the luminosity ranges from $2 \times 10^{41}$ to $10^{42} \mathrm{erg} \mathrm{s}^{-1}$, the effective temperature is about $7 \times 10^{4} \mathrm{~K}$, which peaks at $\lambda \sim 40 \mathrm{~nm}$ (extreme ultraviolet), and the peak occurs about an hour after the nucleosynthesis event. These results are very similar to what Metzger et al. (2015) found, however, they found peak effective temperatures of $\sim 10^{4} \mathrm{~K}$, because they used higher opacities $\left(\kappa=30 \mathrm{~cm}^{2} \mathrm{~g}^{-1}\right)$ since their trajectories still contained a significant amount of lanthanides and actinides (B. Metzger 2015, private communication). In our case, we do not find significant amounts of lanthanides or actinides if we obtain a strong neutron-rich freeze-out, and thus we get a lower opacity, which raises the effective temperature Li \& Paczyński (1998), making such a transient even harder to detect because it peaks deeper in the ultraviolet. It appears that more work is needed to consistently model these neutron-powered transients.

Note that the transition point in $Y_{\mathrm{e}}$ in Figure 9 where the light curve peaks at about $1 \mathrm{hr}$ to where it peaks at a few days is somewhat arbitrary because it depends on how we determine the peak in the light curve. As explained above, we arbitrarily decided to only consider peaks occurring earlier than 0.5 days if they are more than three times brighter than any later peaks. The justification for this is that early peaks are very short and thus hard to detect, but in the cases where we only obtain a short, bright early peak, we do not want to pick out any later peaks that are really just the highest points of very shallow and long plateaus.

We emphasize that the outflows used in this section were assumed to have homogeneous compositions. In nature, outflows from compact object mergers will have some spread in electron fraction and therefore have inhomogeneous compositions. Nonetheless, our simplified models provide guidance on the sensitivity of kilonova light curves to variations in the average electron fraction, entropy, and dynamical timescale during r-process nucleosynthesis.

\subsection{Mass Estimates of Potential Kilonova Observations}

Since the ejecta mass is a parameter in our simplified lightcurve model, we can attempt to put a lower bound on the ejecta mass necessary to reproduce the possible kilonova observations. For the possible kilonova associated with GRB 130603B, there is one observation in the infrared, one upper limit in the optical, and another upper limit in the infrared at late times (Berger et al. 2013; Tanvir et al. 2013). For every point in our low-resolution sym0 parameter space we compute nine light curves with all combinations of $v / c=0.1,0.2,0.3$ and $M / M_{\odot}=0.01,0.05,0.15$. We then compute the observed AB magnitudes that would result from the light curve at the rest frame time when the observations were made, taking into account redshift and the actual filter response of the Hubble Space Telescope (HST) . ${ }^{4}$ Finally, we interpolate the resulting observed magnitudes as a function of the ejecta mass to find the minimum mass that reproduces the observed magnitude in the near-infrared band (HST filter WFC3/F160W, roughly J-band in the rest frame) and produces an optical signal (HST filter WFC3/F606W, roughly B-band in rest frame) that is below the observed upper limits.

We repeat the above procedure for light curves calculated with different heating efficiencies $f$ (see Equation (14)), as the exact value of $f$ is not known but has a direct influence on the brightness of the kilonova. For $f=0.1,0.3$, and 0.5 , we find that the minimum (over our entire parameter space) ejecta masses necessary to match the possibly observed kilonova after GRB 130603B are 0.09, 0.03, and 0.02 solar masses, respectively. This is a reasonable result, as we expect the minimum mass necessary to produce a kilonova of equal brightness to decrease as the heating efficiency increases.

If we repeat the same procedure with the potentially observed kilonova after GRB 060614 (where there are detections in both the near-infrared (Hubble Space Telescope (HST) filter WFPC2/F814W, roughly R-band in rest frame) and optical HST filter WFPC2/F606W, roughly V-band in rest frame), two infrared upper limits at late times, and an optical upper limit at late times (Jin et al. 2015; Yang et al. 2015)), we find that none of our light curves calculated with $f=0.1$ can match the observations, and for $f=0.3$ and 0.5 we require a minimum mass of 0.04 and 0.05 solar masses, respectively. We note that a larger ejecta mass is needed when a larger heating efficiency is assumed. Because there are observations in two bands for GRB 060614, our fits are more sensitive to the spectral temperature found in the light-curve models than in the case of GRB 130603B. Qualitatively, the spectral temperature scales inversely with the mass and proportionally to the heating efficiency (Li \& Paczyński 1998). Therefore, to keep a fixed spectral temperature when increasing the heating efficiency the total mass also must be increased. Our simple method for calculating the effective temperature is likely inadequate for detailed confrontation with multi-band observations, so these minimum masses are necessarily approximate. Another issue with this method of finding the minimum allowed mass is that the outflow does not have a homogeneous composition (e.g., Wanajo et al. 2014; Just et al. 2015; Kasen et al. 2015; Metzger et al. 2015). Thererfore, to acquire more accurate estimates of the minimum ejected mass in these potential kilonova events, more sophisticated light-curve model and hydrodynamical

\footnotetext{
4 http://svo2.cab.inta-csic.es/svo/theory/fps3/index.php? mode $=$ browse $\&$ gname $=$ HST
} 
simulations are required. Such an analysis was performed in Hotokezaka et al. (2013b) for GRB 130603B, where they found preferred ejecta masses between 0.02 and $0.1 M_{\odot}$.

Nevertheless, the work we have presented here will be very useful to estimate the masses and maybe even other parameters from future observations of kilonovae. With a sophisticated radiation transport method, one can calculate accurate light curves using our heating rates and lanthanide and actinide abundances. A consequence of our finding that the heating rate does not strongly depend on $Y_{\mathrm{e}}$ in the lanthanide-rich regime (and not even on $s$ and $\tau$ except at very low $Y_{\mathrm{e}}$ ) is that one will be able to quite accurately estimate the ejecta mass of future observed kilonovae without precisely knowing the values of $Y_{\mathrm{e}}$, $s$, and $\tau$. A caveat is, however, that one needs to know the heating efficiency and lanthanide and actinide opacities well.

\section{CONCLUSIONS}

We have systematically performed nucleosynthesis calculations with our new nuclear reaction network SkyNet for a wide range of three parameters: initial electron fraction $\left(0.01 \leqslant Y_{\mathrm{e}} \leqslant 0.5\right), \quad$ initial entropy $1 k_{\mathrm{B}}$ baryon $^{-1} \leqslant s \leqslant$ $100 k_{\mathrm{B}}$ baryon $^{-1}$, and the expansion timescale $0.1 \mathrm{~ms} \leqslant$ $\tau \leqslant 500 \mathrm{~ms}$ during nuclear burning. We ran the full parameter space with different fission reactions, but found that there were only small quantitative and no qualitative differences between the different fission reactions. We focused our attention on the amount of lanthanides and actinides produced and the heating rate between 0.1 and 100 days after the start of the nucleosynthesis calculation, because kilonova transients are expected to occur in this time frame. With a spherically symmetric, gray radiation transport scheme we estimated the peak time, peak luminosity, and peak spectral temperature of the kilonova light curves.

We find that the final amount of lanthanides and actinides depends most strongly on $Y_{\mathrm{e}}$ and the ejecta is lanthanide-free for $Y_{\mathrm{e}} \gtrsim 0.26$. However, there are some regions of the parameter space where the ejecta is lanthanide-free even for very low electron fractions. Specifically, at high initial entropies and small expansion timescales we get a neutron-rich freeze-out, which does not produce lanthanides, but may result in a very bright, very blue transient on the timescale of an hour. At small initial entropies and very large expansion timescales, there is significant late-time heating, which causes the composition to go back to NSE and effectively restart the r-process at a much higher electron fraction, which was raised by $\beta$-decays.

Since the lanthanides and actinides can increase the opacity of the material by a factor of $\sim 100$, we find that the peak luminosity increases by about one order of magnitude and the light-curve peak timescale goes from about a week to about a day as the ejecta becomes lanthanide-free. This is consistent with previous works by Roberts et al. (2011), Kasen et al. (2013), Tanaka \& Hotokezaka (2013), Grossman et al. (2014). The heating rate at 1 day, however, remains largely unchanged and decreases by no more than one order of magnitude as the ejecta becomes lanthanide-free. Thus the increase in the kilonova luminosity is due to the decrease in the opacity when lanthanides are no longer present, which pushes the peak to earlier times when the heating is stronger. At very high $Y_{\mathrm{e}}$ $(\gtrsim 0.4)$, there are large variations in the heating rate because single nuclides dominate the heating. At lower $Y_{\mathrm{e}}$, the heating rate at 1 day is very uniform in entropy and expansion timescale because it is dominated by an ensemble of nuclides that average out to the same heating rate at 1 day even though the exact composition may be very different. This has already been found in Metzger et al. (2010) and we are now confirming it for a larger parameter space.

Overall, we find only weak correlation between the lanthanide production and heating rate. Both quantities are quite strongly correlated with $Y_{\mathrm{e}}$, but not so much with one another. The heating rate at 1 day is not affected much when the lanthanide abundance suddenly drops by many order of magnitude, but it slowly declines at higher $Y_{\mathrm{e}}$.

In Section 2.4, we provided three linear inequalities involving $Y_{\mathrm{e}}, \ln s$, and $\ln \tau$ that can be used to determine if the ejecta with those properties is lanthanide-rich or lanthanidefree. Those inequalities give the correct answer in $98 \%$ of all cases. We also provide parametric fits for the heating rates between 0.1 and 100 days for all cases at http://stellarcollapse. org/lippunerroberts2015. The mean fractional log difference between the actual heating rate and our fit is no more than $1 \%$ in $95 \%$ of all cases. On the same website, we also provide an integrated fractional heating contribution to give an idea of which specific nuclides contribute the most to the radioactive heating.

Our nucleosynthesis code SkyNet will be released as a free and open-source code soon. In the meantime, those interested can contact the authors about getting early access to the code. Future versions of SkyNet will also include neutrino interactions. Much more work needs to be done to accurately model the light curves of kilonovae and especially to calculate the line structure and hence opacity of the lanthanide and actinide elements. We hope that our heating rate fits will be useful to other researchers to calculate kilonova light curves that could aid with detecting such events.

We thank Dan Kasen for helpful discussions on light-curve modeling and for graciously providing us with temperaturedependent mean opacities for various mixtures of neodymium and iron. We thank Christian Ott for numerous useful discussions and for a careful reading of the manuscript. We thank Brian Metzger for a number of useful comments on the manuscript. And we also thank Shri Kulkarni for discussion about computing observed magnitudes.

The calculations presented here were performed on the Caltech "Zwicky" compute cluster (NSF MRI award No. PHY0960291), on the NSF XSEDE network under allocation TGPHY100033, and on NSF/NCSA Blue Waters under allocation jr6 (NSF PRAC award No. ACI-1440083). Support for L.R. during this work was provided by NASA through an Einstein Postdoctoral Fellowship grant No. PF3-140114 awarded by the Chandra X-ray Center, which is operated by the Smithsonian Astrophysical Observatory for NASA under contract NAS803060. J.L. is partially supported by NSF under award Nos. TCAN AST-1333520, CAREER PHY-1151197, and AST1205732, and by the Sherman Fairchild Foundation.

\section{REFERENCES}

Arcones, A., \& Martínez-Pinedo, G. 2011, PhRvC, 83, 045809

Arcones, A., Martínez-Pinedo, G., Roberts, L. F., \& Woosley, S. E. 2010, A\&A, 522, A25

Argast, D., Samland, M., Thielemann, F.-K., \& Qian, Y.-Z. 2004, A\&A, 416, 997

Barnes, J., \& Kasen, D. 2013, ApJ, 775, 18

Bauswein, A., Goriely, S., \& Janka, H.-T. 2013, ApJ, 773, 78 
Berger, E., Fong, W., \& Chornock, R. 2013, ApJL, 774, L23

Burbidge, E. M., Burbidge, G. R., Fowler, W. A., \& Hoyle, F. 1957, RvMP, 29,547

Chevalier, R. A., \& Soker, N. 1989, ApJ, 341, 867

Cyburt, R. H., Amthor, A. M., Ferguson, R., et al. 2010, ApJS, 189, 240

de Jesús Mendoza-Temis, J., Martínez-Pinedo, G., Langanke, K., Bauswein, A., \& Janka, H.-T. 2014, arXiv:1409.6135

Eichler, M., Arcones, A., Kelic, A., et al. 2014, arXiv:1411.0974

Ensman, L. 1994, ApJ, 424, 275

Fernández, R., \& Metzger, B. D. 2013, MNRAS, 435, 502

Foucart, F., Deaton, M. B., Duez, M. D., et al. 2014, PhRvD, 90, 024026

Freiburghaus, C., Rosswog, S., \& Thielemann, F.-K. 1999, ApJL, 525, L121

Fuller, G. M., Fowler, W. A., \& Newman, M. J. 1982, ApJS, 48, 279

Gehrels, N., Ramirez-Ruiz, E., \& Fox, D. B. 2009, ARA\&A, 47, 567

Goriely, S., Bauswein, A., \& Janka, H.-T. 2011, ApJL, 738, L32

Goriely, S., Bauswein, A., Just, O., Pllumbi, E., \& Janka, H.-T. 2015, arXiv: 1504.04377

Goriely, S., Demetriou, P., Janka, H.-T., Pearson, J. M., \& Samyn, M. 2005, NuPhA, 758, 587

Grossman, D., Korobkin, O., Rosswog, S., \& Piran, T. 2014, MNRAS, 439, 757

Hoffman, R. D., Woosley, S. E., \& Qian, Y.-Z. 1997, ApJ, 482, 951

Hotokezaka, K., Kiuchi, K., Kyutoku, K., et al. 2013a, PhRvD, 87, 024001

Hotokezaka, K., Kyutoku, K., Tanaka, M., et al. 2013b, ApJL, 778, L16

Jin, Z.-P., Li, X., Cano, Z., et al. 2015, arXiv:1507.07206

Just, O., Bauswein, A., Pulpillo, R. A., Goriely, S., \& Janka, H.-T. 2015, MNRAS, 448, 541

Kasen, D., Badnell, N. R., \& Barnes, J. 2013, ApJ, 774, 25

Kasen, D., Fernández, R., \& Metzger, B. D. 2015, MNRAS, 450, 1777

Korobkin, O., Rosswog, S., Arcones, A., \& Winteler, C. 2012, MNRAS, 426, 1940

Kulkarni, S. R. 2005, arXiv:astro-ph/0510256

Langanke, K., \& Martínez-Pinedo, G. 2000, NuPhA, 673, 481

Lattimer, J. M., Mackie, F., Ravenhall, D. G., \& Schramm, D. N. 1977, ApJ, 213,225
Lee, W. H., \& Ramirez-Ruiz, E. 2007, NJPh, 9, 17

Li, L.-X., \& Paczyński, B. 1998, ApJL, 507, L59

Martin, D., Perego, A., Arcones, A., et al. 2015, arXiv:1506.05048

Metzger, B. D., Bauswein, A., Goriely, S., \& Kasen, D. 2015, MNRAS, 446, 1115

Metzger, B. D., \& Fernández, R. 2014, MNRAS, 441, 3444

Metzger, B. D., Martínez-Pinedo, G., Darbha, S., et al. 2010, MNRAS, 406, 2650

Mihalas, D., \& Weibel-Mihalas, B. 1999, Foundations of Radiation Hydrodynamics (Mineola, NY: Dover)

Möller, P., Pfeiffer, B., \& Kratz, K.-L. 2003, PhRvC, 67, 055802

Mumpower, M. R., McLaughlin, G. C., \& Surman, R. 2012, PhRvC, 85, 045801

Nakar, E. 2007, PhR, 442, 166

Nakar, E., \& Piran, T. 2011, Natur, 478, 82

Oda, T., Hino, M., Muto, K., Takahara, M., \& Sato, K. 1994, ADNDT, 56,231

Perego, A., Rosswog, S., Cabezón, R. M., et al. 2014, MNRAS, 443, 3134

Ramirez-Ruiz, E., Trenti, M., MacLeod, M., et al. 2015, ApJL, 802, L22

Richers, S., Kasen, D., O’Connor, E., Fernandez, R., \& Ott, C. 2015, arXiv: 1507.03606

Roberts, L. F., Kasen, D., Lee, W. H., \& Ramirez-Ruiz, E. 2011, ApJL, 736, L21

Rosswog, S. 2013, RSPTA, 371, 20272

Shen, S., Cooke, R., Ramirez-Ruiz, E., et al. 2014, arXiv:1407.3796

Tanaka, M., \& Hotokezaka, K. 2013, ApJ, 775, 113

Tanvir, N. R., Levan, A. J., Fruchter, A. S., et al. 2013, Natur, 500, 547

The LIGO Scientific Collaboration 2015, CQGra, 32, 074001

Timmes, F. X., \& Swesty, F. D. 2000, ApJS, 126, 501

van de Voort, F., Quataert, E., Hopkins, P. F., Kereš, D., \& Faucher-Giguère, C.-A. 2015, MNRAS, 447, 140

Wanajo, S., Sekiguchi, Y., Nishimura, N., et al. 2014, ApJL, 789, L39

Woosley, S. E., \& Hoffman, R. D. 1992, ApJ, 395, 202

Yang, B., Jin, Z.-P., Li, X., et al. 2015, NatCo, 6, 7323 